\title{
I Migrationsgesellschaft: kritische Vistas von Migration, Kapitalismus und Ankommen
}

\author{
Radostin Kaloianov ${ }^{\star}$
}

\section{Zusammenfassung}

Ausgehend von dem Konzept der, Migrationsgesellschaft' und dem daran ansetzenden Versuch, den Migrationsbegriff jenseits geläufiger Engführungen als Wanderung kritisch zu hinterfragen, wird im Folgenden ein umfassender Perspektivenwechsel vorgeführt. Dieser Umbruch geht mit der Kritik an der Idealisierung von Migration als Wanderung und der Verlegung des Fokus von Wanderung auf das Ankommen von MigrantInnen einher. Der Perspektivenwechsel macht am Migrationsthema nicht Halt und setzt sich auch in der Analyse von migrantischem Ankommen fort. Den wanderungszentrierten Szenarien von Niederlassen wie jenes der Integration und des Diversitätsmanagements werden neue Perspektiven auf migrantisches Ankommen entgegengestellt. Setzen diese kritischen Bildgebungen den Fokus aufs Ankommen selbst, zeigen sie, dass das Ankommen von MigrantInnen bei vielfachen Ungleich- und Schlechterstellungen und von unten ansetzt, zahlreiche Ambivalenzen, Widersprüche, Polarisationen aufweist und zugleich ein Niederlassen im Sinne vom nieder Fußfassen lassen ist. Was Migrationsgesellschaft ist und was in der Migrationsgesellschaft los ist, bringt die Formel auf den Punkt: ,Aufs Ankommen kommt es an!'

Schlagwörter: Migrationsgesellschaft, Migration, Kritik, Kapitalismus, Ankommen

\section{Migration society? Critical Perspectives of Migration, Capitalism and Arrival}

Abstract

The article argues for a comprehensive change of perspective, based on the concept of the 'migration society' and the attempt to critically question the concept of migration beyond conventional definitions of migration as wandering. This change of perspective originates in the critique of the idealization of migration as wandering and the shift in conceptional focus from the wandering to the arrival and settling-down of migrants. This paradigm change does not limit itself to the topic of migratory mobility and extends to the analysis of migrant arrival. The wandering-centered scenarios of migrant arrival, such as those of integration and diversity management, are contrasted with new critical understandings of what migrant settlement is all about. Setting the focus on migrant arrival, this approach shows ambivalences, contradictions, and polarizations, that characterize migrant new arrivals as arrivals from below. The point of departure to critically construe migrant settling-down is to take this process literally as a setting a foot down allowing, a settling at the bottom rungs of the social ladder. What migration society is and what is going on in migration society is summed up by the formula: It's all about arriving!

Keywords: migration society, migration, criticism, capitalism, arrival

\footnotetext{
IIIIIIIIIIIIIIIIIIIIIIIIIIIIIIIIIII

* Radostin Kaloianov, Interface Wien/ Universität Hamburg, Institut für Soziologie, E-Mail: r.kaloianov@gmx.at, Tel.: oo4365o 5813480
} 
Der Aufsatz geht der Frage nach, was gemeint sein kann, wenn die gegenwärtigen Gesellschaften Deutschlands oder Österreichs als, Migrationsgesellschaften' bezeichnet werden. Die Beantwortung dieser Fragestellung artikuliert und argumentiert die kritische Erkenntnis, dass es in Migration und bei jenen, die als MigrantInnen gelten, vor allen Dingen auf das Ankommen ankommt und eine Migrationsgesellschaft im Kern als Ankunftsgesellschaft zu verstehen ist. Im Einzelnen befassen sich die Argumentationsschritte 1) mit Migrationsgesellschaft als gesellschaftstheoretische Perspektive, die sich vor dem Hintergrund 2) vorherrschender wissenschaftlicher wie außerwissenschaftlicher Bezugnahmen auf Migration mit Fokus auf Wanderung herausbildet. Daraufhin wird nachgezeichnet, wie das Wanderungsmodell der Migration kritisch hinterfragt und überschritten werden kann sowohl in wissenschaftlichen Theorien zur 3) Migrationsgesellschaft und insbesondere zur 4) Postmigration als auch 5) in fachlichen und alltäglichen Adressierungen von Menschen als MigrantInnen. Auf diese Weise wird auch dem umgreifenden Paradigmenwechsel nachgegangen, der in der Theorie und Praxis von Migration den Fokus von der Wanderung auf das Ankommen verschiebt und so auch den Weg für eine 6) full vision von Migration als Nexus von Wanderung und Ankommen öffnet.

Abschließend sollen die wichtigsten Bausteine einer kritischen Theorie von migrantischem Ankommen umrissen werden. Diese setzt sich 7) von hegemonialen Konzepten von Integration und Diversitätsmanagement mit ihrer unterschwelligen Fokussierung auf Wanderung kritisch ab und 8) versteht migrantisches Ankommen jenseits von Wanderung und mit Fokus auf das Fußfassen und Niederlassen als Ankommen von unten. Dieses Ankommen von unten in big numbers ist die Haupthandlung, die sich auf den westlichen Bühnen des gegenwärtigen Kapitalismus abspielt und diese zu den 9) Ankunftsgesellschaften oder Postwanderungsgesellschaften macht, die Migrationsgesellschaft als kritische Perspektive im Sinn und vor Augen hat.

\section{Die Perspektive: ,Migrationsgesellschaft}

In Theorie und Praxis wird des Öfteren auf Migrationsgesellschaft als ein Platzhalter verwiesen, in dem man verschiedene Praxisfelder wie Sozialarbeit (Otto/ Schrödter 2006) und wissenschaftliche Disziplinen wie etwa Pädagogik (Mecheril 2004) platzieren kann.
Die Migrationsgesellschaft wird für einen empirisch feststellbaren Gesellschaftszustand gehalten, der bestimmte Kriterien der Transformation und Sättigung durch Migration, der Offenheit oder Verschlossenheit gegenüber MigrantInnen erfüllt und aus diesem Grund als migrationsgesellschaftlich bezeichnet wird. Was uns ermöglicht, Migrationsgesellschaft als empirisches Objekt zu erfassen, sind aber weder nur die empirischen Fakten der Migration noch ausschließlich die normativen Maßstäbe ihrer Auslegung oder die gesellschaftlichen Transformationen durch Migration, sondern die Perspektive, welche Fakten, Normen und Veränderungen überhaupt erst für Indizien der Migrationsgesellschaft hält und diese in einem Zusammenhang betrachtet. So bezeichnet ,Migrationsgesellschaft' an erster Stelle eine Perspektive auf reale Adressen und Zustände von Gesellschaften, die mit Migration zu tun haben, sich durch Migration verändern, aber auch umgekehrt Prozesse und AkteurInnen der Migration verändern. Eigentlich können diese empirischen Adressen, an denen Migrationsgesellschaft zu Hause ist, gar nicht gemeldet, notiert und gefunden werden, ohne dass eine gewisse Perspektive diese kartiert.

Ursprünglich wird ,Migrationsgesellschaft ${ }^{\star}$ in Deutungskämpfen als kritisches Konzept eingeführt, das die Thematisierung von Migration jenseits von populären Engführungen als Wanderung oder Einwanderung ermöglicht. Letzteres ist in öffentlichen Bildgebungen und Leitkonzepten einer Aufnahmegesellschaft der Fall, die sich als ,Einwanderungsland oder ,Einwanderungsgesellschaft' begreifen will. Die Fortschrittlichkeit gesellschaftlicher Selbstbilder auf Einwanderung auszurichten, von einem „informellen“ zu einem „formellen modernen Einwanderungsland" (Rahner 2011: 32) voranzuschreiten und Einwanderung als prägende Rahmung der Gesellschaft anzuerkennen, wird gewürdigt. ${ }^{1}$ Zugleich wird aber kritisiert, dass diese Fortschrittlichkeit nur vor dem Hintergrund von Rückständigkeit und eines rückwärtsgewandten Umgangs mit Migration auffällt und eigentlich einen Fortschritt darstellt, der weiterhin den Entwicklungen massiv hinterherhinkt.

Zum anderen spiegelt die Namensgebung des Konzepts wissenschafts-disziplinäre Interessen und Ansprüchen wider, Forschungsterrain, Gegenstandsfeld, Epistemologie und Methodologie der Migrationspädagogik (Mecheril et al. 2016) abzustecken. Was

1 vgl. Mecheril 2016: 13 . 
dabei ins Gewicht fällt, ist weniger sich gegenüber anderen, angrenzenden pädagogischen Richtungen abzugrenzen. Vielmehr geht es darum, der Migrationspädagogik mit dem Begriff der Migrationsgesellschaft eine Art ontologisches Korrelat vorzulegen, zu markieren, wo die Migrationspädagogik verortet ist, worüber und worum es sich bei ihr handelt: Die Migrationspädagogik ist Pädagogik in, über und für eine Gesellschaft, die sich uns diagnostisch als Migrationsgesellschaft darstellt. Als Pädagogik, welche die Gesamtgesellschaft als Migrationsgesellschaft anvisiert und zum eigenen Forschungsterrain und Erkenntnisgegenstand beansprucht, nimmt sich die Migrationspädagogik Aufgaben und Themen vor und erhebt Ansprüche, die über die Aufgaben, Themen und Ansprüche herkömmlicher Pädagogik hinausgehen. In diesem big shot, auf das große Ganze losgehen zu wollen, steckt der programmatische Anspruch, sich nicht auf die Rolle einer pädagogischen Nischen-Disziplin reduzieren zu lassen, den eigenen Wirkungsradius nicht auf die MigrantInnen als Nischen-Gruppe und Nischenthema, auf Migration als bloße ,Querschnittmaterie‘ zu beschränken oder schlichtweg nicht das nächste „migrantologische“ Fach zu werden (Bojadžijev/Römhild 2014: 1off.). Vielmehr ist der Anspruch, aus dem Fokus auf Migration einen Erkenntnisvorteil zu beziehen, sich durch Migration als Methode in eine privilegierte Erkenntnisposition zum großen Ganzen der Gesellschaft zu bringen, um durch das Prisma der Migration gesamtgesellschaftliche Veränderungen anders und kritisch begreifen $\mathrm{zu}$ können.

Als Perspektive darauf, was mit Migration im Aufeinandertreffen mit Gesellschaft passiert, und umgekehrt, wie sich dieses Aufeinandertreffen auf westliche Gegenwartsgesellschaften auswirkt, was und wie sehr es diese verändert und eben in Migrationsgesellschaften verwandelt, reagiert die migrationsgesellschaftliche Perspektive unmittelbar auf verschiedene perspektivische Fehlgriffe. Letztere laufen darauf hinaus, MigrantInnen in den mehrheitsgesellschaftlichen Repräsentationsordnungen, Erkenntnissystemen und Wirklichkeitsdeutungen verkehrt, halbiert, negiert oder gar nicht so ankommen zu lassen, wie sie bereits in Wirklichkeit angekommen sind. Als Kritik bringt sich die migrationsgesellschaftliche Perspektive in den sich daraus ergebenden Deutungskämpfen aktiv ein, schließt aufklaffende Erkenntnislücken, repariert Bildzerrungen und weist selbst auf die Widersprüche des migrantischen Ankommens hin, wie etwa auf den Widerspruch zwischen der realen wirtschaftlichen, gesellschaftlichen Unentbehrlichkeit von MigrantInnen und „der kulturell-politischen Logik der Abschottung“ (Bojadžijev et al. 2018: 8)² gegenüber Migration in Ländern wie Deutschland oder Österreich. ${ }^{3}$

Zwei der am meisten bekannten und folgenreichen Weisen, in fachlichen und öffentlichen Bildgebungen MigrantInnen verkehrt oder jedenfalls nicht so ankommen zu lassen, wie sie in Wirklichkeit bereits angekommen sind, sind MigrantInnen a) als nonstopWandernde anzusehen, und b) das migrantische Ankommen hauptsächlich von außen zu erwarten.

Verkehrt ist die erste gängige Bildgebung von Migration und MigrantInnen, weil sie migrantisches Ankommen wie ein Kommen-Gehen-Kommen-Gehen ... ohne Ende-Karussell und nicht wie ein ,Ankommen, um zu bleiben' porträtiert. Sich MigrantInnen so gänzlich verkehrt als Kommer-GeherInnen vorzustellen, die keine Spuren hinterlassen und angeblich möglichst viel erwischen und wegtragen würden, macht es unmöglich, MigrantInnen als das zähe Bodenpersonal zu erkennen, welches gekommen ist, um zu bleiben, ein neues Zuhause errichten und etwas Bleibendes hinterlassen will.

Auch die nicht weniger verbreitete und einflussreiche Vorstellung, dass Migrationen und MigrantInnen von außen herkommen, lässt MigrantInnen verkehrt in den tonangebenden Auffassungen über Migration ankommen. MigrantInnen mögen zwar von woanders herstammen und von außen einwandern, jedoch kommen jene, die als MigrantInnen gelten, von einem Außen her, das gegenüber den westlichen Ankunftsarenen ungleich, schlechter und unterhalb gestellt, somit mehr unten als außen ist, also fassen

2 „Widerspruch zwischen dem ökonomischen Bedarf an migrantischen Arbeitskräften einerseits und der kulturell-politischen Logik der Abschottung andererseits. Was ist damit gemeint? Der ökonomische Bedarf an migrantischer Arbeitskraft schwankt zwar konjunkturell, war aber in den meisten Phasen seit Bestehen der Bundesrepublik ein wichtiger Faktor für die Migrationspolitik. Demgegenüber steht eine verbreitete politische und kulturelle Logik, die Einwanderung im Allgemeinen skeptisch bis ablehnend gegenübersteht und die Migration in die Bundesrepublik möglichst einschränken oder komplett verhindern möchte“ (ebd.).

3 In Cem Karacas Lied „Es kamen Menschen an“ klingt dieser „Gastarbeiter-Widerspruch“ (Fischer 2020: 31) so: „Man brauchte unsere Arbeitskraft,/die Kraft, die was am Fließband schafft ...Wir Menschen waren nicht interessant, darum blieben wir euch unbekannt“ (Karaca 2010). 
MigrantInnen Fuß zunächst von unten. Ihr Niederlassen fängt chronologisch von vorne, geografisch von außen, gesellschaftskritisch betrachtet aber von unten an.

Die migrationsgesellschaftliche Perspektive übt sich in perspektivischer Schubumkehr gegenüber verbreiteten kopfübergestellten, halbierten, karikierten, negierenden Vorstellungen von MigrantInnen in den aufnahmegesellschaftlichen Bildgebungssystemen.

\section{Migration: Fokus auf Wanderung und Idealisierung als Wanderung}

In wissenschaftlichen Definitionen kommen besonders klar die grundlegendsten Komponenten der Erkenntnis und des Verständnisses von Migration hervor, was sie zu besonders ergiebigen Spuren für die vorherrschende Fokuslegung auf Wanderung macht. Dies ist zutreffend sowohl für das Wissen über Migration, das man als niedergelassen bezeichnen kann und das sich im wissenschaftlichen, aber auch im breiteren Mainstream re-produziert, als auch für das fluide, im Entstehen, im Streit oder in Diskussion begriffenes Wissen der Forschung.

Mit welchem Fokus an Migrationsphänomene herangegangen wird im Bereich des niedergelassenen Wissens, das durch Einrichtungen der Politik, der Verwaltung, der Zivilgesellschaft, der akademischen Lehre, der anwendungsorientierten Forschung konsumiert und zugleich reproduziert wird, lässt sich primär und gezielt aus Quellen wie Lehrbüchern, Enzyklopädien, Lexika, Positionspapieren, Leitbildern oder Medienberichten herauslesen.

In den Recommendations on Statistics of International Migration (UN DESA 1998) definiert das United Nations Department of Economic and Social Affairs (UN DESA) „long-term migrant“ als „a person who moves $[\mathrm{mH}]$ to a country other than that of his or her usual residence for a period of at least a year" (UN DESA 1998: 10). Diese Grundlagenkonzeption legt den weltweiten Standard vor, was als Migration zu verstehen ist. Die zitierte Definition wird in leicht variierenden Ausformulierungen in verschiedenen Erkenntniskontexten und durch verschiedene internationale, nationale und lokale Institutionen übernommen. Wortwörtlich wird die besagte UNDefinition von „long-term migrant" durch die OECD übernommen, wo ebenso festgehalten wird, dass „the term ,migrant ${ }^{`}$ generally functions as an umbrella term used to describe people that move $[\mathrm{mH}]$ to ano- ther country“ (OECD 2018: 26). Letztere Definition besagt, dass nicht nur MigrantInnen Personen sind, die wandern, sondern auch die grundlegenden Konzepte und Definitionen über MigrantInnen travelling concepts sind, die sich meistens unverändert in den Fachvokabularen internationaler Institutionen vorfinden, denn auch für das Glossar der International Organization for Migration vom 2019 ist „migrant ... an umbrella term, not defined under international law, reflecting the common lay understanding of a person who moves $[\mathrm{mH}]$ away from his or her place of usual residence, whether within a country or across an international border, temporarily or permanently, and for a variety of reasons" (IOM 2019a: 132). ${ }^{4}$

An denselben Standard, Migration und MigrantInnen mit Fokus auf Wanderung zu verstehen, hält sich - und bekräftigt ihn dadurch zusätzlich - auch der durch seine Vielfalt an Methoden, Datenquellen, AutorInnen, Expertisen und Themen in etlicher Hinsicht weltweit maßgebende Forschungsbericht zur Migration, der World Migration Report (WMR) der International Organization for Migration (IOM). Der WMR wird vom Standpunkt einer wanderungsfokussierten Perspektive auf Migration verfasst, worauf das klare Bekenntnis zu den Standard-Definitionen (siehe die Zitate aus UN DESA 1998 und IOM 2019a oben) hinweist (IOM 2019b: 2of.).

Noch deutlicher kommt das wanderungszentrierte Grundverständnis von Migration hervor, wenn man den World Migration Report einer elementaren diskursanalytischen Prüfung unterzieht und ihn nach Schlüsselbegriffen hin untersucht, die jeweils dem semantischen Spektrum der Mobilität bzw. des Ankommens zuzuordnen sind. Das Ergebnis ist eindeutig: Für die Erstellung der weltweit umfangreichsten Bildgebung von Migration wird von mehr als doppelt so vielen Schlüsselbegriffen, die auf Wanderung („move“, „mobility“, „travel“ = 441 Treffer) verweisen, als solchen, die Ankommen thematisieren („stay“, „settle“, „arrive“, „arrival“ = 211 Nennungen), Gebrauch gemacht. ${ }^{5}$

4 „MigrantIn“ wird also durch die IOM als „ein völkerrechtlich nicht definierter Überbegriff“ ausgewiesen, „der das gängige Laienverständnis einer Person widerspiegelt, die sich von ihrem gewöhnlichen Aufenthaltsort entfernt, sei es innerhalb eines Landes oder über eine internationale Grenze, vorübergehend oder dauerhaft und aus einer Vielzahl von Gründen“. (eigene Übersetzung).

5 In einem ähnlichen Verhältnis stehen die Fokusse auf Wanderung und Ankommen in seminalen Nachschla- 
Ähnlich wie im Mainstream-Wissen, das durch wissenschaftliche, politische, internationale Institutionen über Migration im Umlauf gehalten wird, sind die Fokusse auf Wanderung bzw. Ankommen asymmetrisch auch in den Grundannahmen verteilt, auf welche sich die Produktion des neuen und fluiden Wissens über Migration im Feld der Grundlagenforschung stützt. Wiederholt man die oben vorgeführten diskursanalytischen Stichproben an bahnbrechenden Werken der gegenwärtigen Migrationsforschung, ergeben diese ein ähnliches, auf Wanderung fokussiertes Grundverständnis von Migration. In The Postcolonial Age of Migration bemüht Ranabir Samaddar (2018) mehr als fünfmal (!) so viele Schlüsselbegriffe aus dem Bedeutungsregister der Mobilität, als er von Konzepten mit Fokus auf das Ankommen Gebrauch macht. ${ }^{6}$ Auch in dem einflussreichen Standardwerk von Stephen Castles und Mark Miller The Age of Migration (1998), das The Postcolonial Age of Migration kritisch repliziert, kommen wanderungszentrierte Schlüsselkonzepte (wie „mobility“, „mobile“, „move“, „movement(s)“, „flow(s)“, „travel“) um mehr als 50 Prozent häufiger als Begriffe vor, die um den semantischen Kern des Niederlassens gravitieren („settle“, „settlement“, „arrive“, „arrival(s)“, „stay(ing)“, „accommodation“). Die Diskursanalyse in programmatischen wie paradigmatisch wichtigen Texten und Werken der zeitgenössischen Migrationsforschung belegt unmissverständlich die Bevorzugung von Wanderung als Fokus zum Definieren, Thematisieren, Analysieren, Explorieren von Migration. Die Bevorteilung von Wanderung im Auffassen von und Befassen mit Migration ist allgegenwärtig und gehört zu den (wenigen) unhinterfragbaren Grundannahmen in der Erforschung von Migrationsphänomenen: "Migration involves movement; migrants move“ (Griffiths et al. 2013: 10).

gewerken, die den Stand der internationalen akademischen Migrationsforschung abbilden. In der Anthologie Migration (Anderson/Keith 2014) übertreffen die Begrifflichkeiten aus dem semantischen Spektrum der Wanderung (wie "move“, "movement", „mobile“, „mobility“, „travel“, „flow“) mit insgesamt 519 Nennungen mehr als 2,5-mal die Begrifflichkeiten aus dem semantischen Spektrum des Ankommens (wie „arrive“, „arrival“, „stay“, „settle“, „settling“, „accommodate“) mit ihren insgesamt 195 Nennungen.

6 In diesen Fall stehen den wanderungsaffinen Konzepten (wie „mobility“, „mobile“, „move“, „movement(s)“, „flow(s)“) mit insg. 356 Nennungen 67 Treffer für Begriffe mit Ankommensbezug („,stay“, „arrive“, „arrival(s)“, „settle“, „settlement") gegenüber.
Der Fokus auf Wanderung wird auch inter- und transdisziplinär getragen. In so unterschiedlichen disziplinaren Feldern wie Geografie, Soziologie (Treibel 2008), Pädagogik (Hummrich/Terstegen 2020), Migrationspädagogik (Oberlechner 2018) sowie auch der Staatsbürgerschaftstheorie (Bauböck 2006) oder der Grenzregimeforschung (Hess et. al. 2016) wird auf verschiedene Themen, Aspekte, Phänomene von Migration mit Fokus auf Wanderung herangegangen.

Auch zentrale Kategorien der Migrationsforschung und Dimensionen des Phänomens der Migration wie Raum (Faist 2006), Zeit (Bastian 2014), die Transnationalität (Glick-Schiller 2018) und die Geschichte (Hoerder 2014) von Migrationen, staatliche Grenzen und „Grenzregimen“ (Hess/Kasparek 2010) oder auch das „making migration“ (Tazzioli 2019) werden hauptsächlich durch das Prisma von Wanderung und/oder Mobilität betrachtet, definiert und untersucht. Der omnipräsente Fokus auf Wanderung macht auch vor der Thematisierung von Gesellschaft im Auftreffen mit Migration nicht Halt, biegt das Gesellschaftsbild in Richtung Wanderung. „Einwanderungsland“ (Rahner 2011: 32) und „Einwanderungsgesellschaft" (siehe dazu kritisch Mecheril 2016: 13) lauten die relevanten Leitkonzepte.

Wie weiter unten gezeigt wird, erstreckt sich die Fokussetzung auf Wanderung auch im Erfassen von Abschnitten und Handlungszusammenhängen von Migration und Ankommen, die nach der Wanderung folgen, also „post“-Wanderung, „Postmigration“ sind. Hegemoniale politische Narrative und national-staatliche Imperative über die „Integration“ von MigrantInnen (Bojadzjiev 2018) sind das bekannteste Beispiel dafür, wie der allgegenwärtige Fokus auf Wanderung auch das fachliche und populäre Verständnis und die Bildgebungen von migrantischem Ankommen formt. Das Konzeptualisieren von Ankommensprozessen und -realitäten als „Integration“ setzt die Akzente auf das An-Halten einer ausklingenden Wanderung.

Die Gründe dafür, dass der Fokus auf Migration überwiegend auf Wanderung gelegt wird, ausführlich $\mathrm{zu}$ analysieren, könnte das Thema einer eigenständigen Studie sein. Zur Einführung wird hier nur auf einen zentralen Erklärungsansatz wanderungszentrierter Konzeptualisierungen von Migration eingegangen. Der Wanderungsfokus geläufiger Thematisierungen von Migration erfolgt daraus und dann, wenn Migration gesellschaftstheoretisch dekontextualisiert und stattdessen - mehr oder weniger kenntlich - natur- und exaktwissenschaftlich kontextualisiert wird, also mit 
physikräumlichen Bezugsgrößen, naturlandschaftlichen Elementen, naturgeografischen und politischgeografischen Objekten in Bezug gesetzt wird. ${ }^{7}$ Wie bekannte Bezeichnungen von ,Migrationsströmen, ,Flüchtlingswellen', ,Migrationsrouten ' usw. zu verstehen geben, gehen die Bespiegelungen von Migration in naturlandschaftlichen Gegebenheiten an den theoretischen Auffassungs- und Darstellungsweisen nicht spurlos vorbei. Im ,Spiegel der Natur' widergespiegelt, werden den Prozessen, Arten und AkteurInnen von Migration naturlandschaftliche Morphologien und Toponyme aufgetragen, sie werden danach getauft, (nicht nur) metaphorisch mit Naturobjekten verglichen, sondern auch für solche wortwörtlich gehalten. Wenn sich beispielsweise ,Migrationsströme ${ }^{\text {(8) }}$ (Ströme als naturphysische Objekte) durch die „Balkanroute“ durchschlängeln (Balkan ist die türkischsprachige Bezeichnung einer Gebirgskette der Balkanhalbinsel), sind die Migrationen und all das, was sie ausmacht, mit den entsprechenden Naturlandschaften verwachsen. Migrationen werden dabei im Zuge und in Folge ihrer quasi-naturwissenschaftlichen Kontextualisierung nicht nur an naturlandschaftlichen Objekten bespiegelt, sondern werden selbst in naturlandschaftliche Objekte verwandelt, als solche bezeichnet und auch als solche behandelt, als müssten Naturgewalten gewaltvoll (durch Mauern, Zäune, Sperren) eingedämmt oder technologisch (z. B. durch biometrische Identitätsfeststellungen, Knaut 2013) überlistet werden.

Migrationsthemen werden in naturlandschaftliche Bezugssysteme eingepasst und in exaktwissenschaftlicher Manier in diesen Bezugssystemen beforscht: Mengen (der Wandernden) berechnen, Messungen (von Streckenlängen bis zu Wohlstandsgefällen) vornehmen, Daten (über Beweggründe, Bewegungsarten,

7 Auch wenn die Wissenschaft einen maßgeblichen Anteil an der Entstehung und Etablierung des Wanderungsmodells von Migration hat, kann die Überbetonung von Wanderung in Migrationsphänomenen auch auf andere, außerwissenschaftliche Quellen zurückgeführt werden. So lenken Steigerungslogiken kapitalistischer Wertschöpfung tendenziell die Aufmerksamkeit auf Aspekte von Mobilität, die zum Erzielen von Wachstum ausschlaggebend sind und auch Migrationsphänomene mehr in den Fokus der Wanderung rücken.

8 Auch „in the postcolonial age of migration“ sind die "mixed and massive ... population flows" (Samaddar 2018: 8) Hauptgegenstand der (kritischen) Migrationswissenschaft.

9 Schwiertz/Ratfisch 2015, bordermonitoring.eu (2015)
Bewegungsmittel, Routen usw.) sammeln, Koordinaten und Richtungen (von Migrationsströmen oder Flüchtlingswellen) aufzeichnen, all das stellt die Wanderung in verschiedensten Modifikationen ins Rampenlicht und befeuert zusätzlich die Idealisierung von Migration als Wanderung.

Die Überfokussierung von Wanderung in wissenschaftlichen Bildgebungen von Migration ist engstens mit dem exaktwissenschaftlichen Erkenntnisinteresse verflochten, vor der Folie von natur- und politikgeografischen Bezugssystemen, Migration quantifizieren und vermessen $\mathrm{zu}$ wollen. Es werden daher messbare raum-zeitliche Aspekte und Koordinaten aufgegriffen, was das Wandern, den Orts- und Positionswechsel im Raum zum ersten Anwärter macht, stellvertretend für das gesamte Phänomen der Migration untersucht $\mathrm{zu}$ werden. Das Wandern sticht sozusagen ins Auge, wenn man sich dem Thema mit dem Interesse annähert, Migrationen auf ähnliche Weise $\mathrm{zu}$ berechnen und zu vermessen wie die Naturlandschaften und Staatsterritorien, welche die migratorischen Wanderungen durchqueren.

Die besprochenen naturlandschaftlichen Kontextualisierungen und exaktwissenschaftlichen Verarbeitungen von Migration ergeben eine Idealisierung von Migration als Wanderung in zwei sich aufeinander zubewegenden Schritten. Migration als Wanderung zu idealisieren, heißt a) Migration nicht als das auszugeben, was Migration ist - nämlich ein Nexus von Wandern und Niederlassen - und umgekehrt b) Migration für das zu halten, was Migration nicht (nur) ist - nämlich Wanderung.

Migration nicht als das auszugeben, was sie ist, ergibt Idealisierung durch die Reduzierung, Vereinseitigung, Vereinfachung von Realität und kann dazu führen, Realitäten der Migration, die nichts mit Wanderung zu tun haben, durch die idée fixe der ,Wanderung zu unterschätzen, zu verkennen oder gänzlich auszublenden. In dieser Reduzierung und vereinseitigenden Darstellung steckt aber bereits auch der umgekehrte Gedankenzug, Migration ausschließlich für die Wanderung zu halten, die nicht (allein) Migration ist. In diesem zweiten Schritt wird Migration auf eine Art idealisiert, die Konzepten von ,Ideal' und ,Idealisierung' näher kommt. Migration wird als Wanderung idealisiert, wenn Wanderung in Übergröße, überzogen, übertrieben und überbewertet dargestellt wird.

Migration als Wanderung zu idealisieren, heißt nicht Wanderung zum exklusiven oder überwiegenden Thema, sondern zum exklusiven oder überwie- 
genden Fokus zu machen, der in all den vielfältigen Herangehensweisen, Themen und Fragestellungen von Migrationsforschung bis zur Migrationspolitik eingenommen wird. Im Wanderungsfokus überlagern sich die Fluchtpunkte von ansonsten sehr unterschiedlichen Erkenntnisbildern zu Migration. Wanderung ist die Erstannahme, die man über Migration macht und die auch überall explizit oder implizit mitschwingt, wo über Migration geforscht, gelehrt, politisiert, administriert, gesprochen, diskutiert usw. wird.

Der Wanderungsfokus fungiert wie ein unsichtbarer Faden, der sich quer durch die verschiedensten Themen und Zugänge zur Migration hindurchzieht und diese in einem gesamten Modell zusammenschnürt. Im Wanderungsparadigma der Migration bildet Wanderung jenen unauflösbaren Restgehalt des Migrationsphänomens, der bei aller thematischen Varietät versichert, dass es sich um Migration handelt. In diesem Sinne als Erkenntnisfokus verstanden, liefert Wanderung die unabdingbare Prämisse dafür, Migration überhaupt thematisieren zu können und zum Gegenstand von Wissenschaft, Politik, Verwaltung, von Sprechen und Handeln, von Aktivismus und Kritik usw. machen zu können. Diese fokale, nicht thematische Priorisierung von Wanderung in den diversen theoretischen wie praktischen Zugängen zur Migration fällt gerade bei jenen Migrationsthemen am deutlichsten auf, die mit Wanderung rein gar nichts zu tun haben.

Wie lässt sich nun die Idealisierung von Migration als Wanderung kritisch entzaubern?

\section{Kritik und Paradigmenwechsel: von Wanderung} zum Ankommen.

So wie die Idealisierung von Migration auf die zwei ineinandergreifenden Momente der Reduzierung und Übertreibung als Wanderung zurückgeht, belaufen sich auch die Möglichkeiten der Kritik auf zwei darauf reagierende Schritte kritischer Entgegnung.

Die erste Richtung kritischer Analyse nimmt die Übertreibung und Überfokussierung von Wanderung ins Visier, holt das unantastbare Idealbild von Migration als Wanderung auf den Boden der Realität, konfrontiert es mit Alternativen darüber, was Migration noch ist, was noch als Migration zählt und zur Migration dazugehört. Zwei Aspekte machen hier das genuin kritische Profil dieses ersten Kritikschritts aus (Kaloianov 2019). Einerseits wird wider Erwarten Wanderung als Alleinstellungsmerkmal von Migration demontiert und deren Überbesetzung und Übertreibung herunter- gefahren, was bereits die Ersteinführung des diagnostischen Konzepts von „Migrationsgesellschaft“ (Mecheril 2004) anstößt. Andererseits wird insbesondere in theoretischen Perspektiven auf „Postmigration“ die idealisierende Übertreibung von Migration als Wanderung auf den Kopf gestellt und der Fokus auf etwas anderes als Wanderung, auf das Niederlassen oder das Ankommen, das „Nach der Migration“ (Ylldı/Hill 2014), nach der als Wanderung idealisierten Migration verlegt.

Dies ermöglicht und leitet bereits den zweiten Kritikschritt ein, nach der Destabilisierung der Übertreibung von Wanderung und nach der Fokusumkehrung auf das Ankommen auch den zweiten Moment der Idealisierung, die Reduzierung von Migration auf Wanderung zurückzustellen und eine full vision auf die Realität von Migration als Nexus von Wandern und Niederlassen herzustellen. Mit dem Erlangen einer full vision auf die Realität von Migration, in ihrer Gesamtheit und nicht reduziert auf Wanderung, geht das Kritische dieses Schritts nahtlos in den Realismus über, die Vereinseitigung und Übertreibung von Migration als Wanderung rückabzuwickeln und im Zusammenhang von Wanderung und Niederlassen einen Blick auf die komplettierte und entidealisierte Realität von Migration zu bekommen.

Durch die Einführung des Konzepts der Migrationsgesellschaft (Mecheril 2004) vollzieht die Migrationspädagogik den ersten der beiden Schritte der Kritik an der Idealisierung von Migration als Wanderung. Das strategische Interesse der Migrationspädagogik, nicht in die Ecke abgedrängt $\mathrm{zu}$ werden und sich hauptsächlich mit Ein-Wandernden oder frisch EinGewanderten befassen zu müssen, nimmt nicht zufällig Kurs auf eine Kritik, welche die Übertreibung von Migration als Wanderung in Frage stellt und sich gezielt gegen die Über-Betonung und Überfokussierung auf Ein-Wanderung wendet. Mit, Einwanderung' wird eine Spitzenform und Schlüsselphase von Wanderung stellvertretend für das Gesamtphänomen von Wanderung überfokussiert und überzogen dargestellt und ebenso stellvertretend für die Idealisierung von Wanderung als Migration entzaubert. ${ }^{10}$

10 Mecheril 2016: 13. Die Leitrolle des Einwanderungskonzepts im konzeptuellen Spektrum des ,Wanderns, die Überbesetzung von Ein-Wanderung als Wanderungsmodalität und Wanderungsphase geht selbst in entscheidendem Maße auf ihre Beanspruchung durch staatliche, mediale, zivilgesellschaftliche Bildgebungen über den aufgeschlossenen und liberalen Umgang der deutschen Gesellschaft mit Migration zurück.
I/IOMENTUM QUARTERLY (IIIIIIIIIIIIIIIIII 105 
Wie sich aber herausstellt ist das, was das Konzept von ,Migration' mehr und anders als ,Einwanderung oder ,Zuwanderung ' umfasst und was durch die Übereinblendung der letzten beiden verloren geht, selbst nichts anderes als Wanderung und andere Modi von Wanderung (wie „Transmigrationsprozesse“, Mecheril 2016: 14f.). Die kritische Horizonterweiterung über Einwanderung oder Zuwanderung hinaus bewegt sich hier zwar noch innerhalb des Horizonts der Wanderung, dennoch bleibt in diesem first try, in dieser ersten Spur hin zur kritischen und realistischen Vista ${ }^{11}$ von Migration, nichts beim Alten und nichts das Gleiche.

Von dieser Binnendemontage des Wanderungsmodells der Migration ausgehend, ist es nicht so schwierig, den Schritt über das migrationstheoretische Wanderungsparadigma hinaus $\mathrm{zu}$ machen und in Migration etwas anderes als Wanderung zu sehen. „Im Unterschied zu dem Begriff Einwanderung handelt es sich bei Migration um einen analytischen Terminus, der breiter aufgestellt ist, da er nicht lediglich Wanderungsbewegungen von Menschen von Station A nach B beschreibt. Vielmehr beschäftigt sich der Begriff Migrationsgesellschaft mit den durch Wanderungsbewegungen ausgelösten Veränderungsprozessen innerhalb der Gesellschaften“" (Foroutan/Ikiz 2016: 138).

Verlässt man aber das Wanderungsmodell, stellt sich die Aufgabe kritischer Bildgebung von Migration anders und positiv, nämlich aufzeigen zu können, was Migration ist, wenn und weil diese nicht, nicht nur und nicht hauptsächlich Wanderung sein kann.

\section{Postmigration ist Ankommen}

Den positiven Befund von etwas anderem als Wanderung, das man der Idealisierung - Übertreibung und Reduzierung - von Migration als Wanderung entgegensetzen und so das Wanderungsparadigma zur Gänze verlassen und überschreiten kann, machen Theorien und Studien zur „Postmigration“. ${ }^{12}$

11 Der Begriff, Vista' wird hier gleichbedeutend mit Ausblick verwendet und bezeichnet die kritisch-theoretische Bildgebung von Migration und Migrationsgesellschaft, welche aus der Fokusverlegung von Wanderung aufs Ankommen hervorgeht und wider Erwarten vorherrschende Idealisierungen besagter Themen auf den Kopf stellt, also ein zugleich kritischer und realistischer Ausblick bietet. Für einen sinngemäß ähnlichen Gebrauch von ,Vista' siehe Mezzadra (2020: 431).

12 Weiterführend zur „postmigrantischen Perspektive" (Yildız 2019), siehe auch Foroutan 2019; Ylld1z/Hill 2014 2019; Foroutan et al. 2018.
Dieser neue Forschungsstrang untersucht die Migration „nach der Migration“ (Yıldı/Hill 2014), dreht also das Wanderungsmodell um 180 Grad. Im Niederlassen, welches post, nach der Wanderung kommt, aber auch wider die Wanderung steht und als Gegenpol zur Wanderung wirkt, findet dieses Konzept den Anhaltspunkt, um die Idealisierung von Migration als Wanderung durch einen positiven Gegenvorschlag entzaubern zu können. Der Begriff der Postmigration ist der Fachausdruck dafür, was allgemeinverständlich als Niederlassen oder Ankommen bezeichnet wird.

Das „Ankommen auf eigene Rechnung“ ist „Teil einer postmigrantischen Praxis“ (Y1ld1z 2014: 25) und zentrale Lebensrealität in der Migrationsgesellschaft und im gesamten Migrationsprozess, der sich faktisch nur zu einem kleinen, zeitlich kurzen Teil tatsächlich als Wanderung vollzieht. In postmigrantischen Perspektiven erhält diese Realität die Vorrangstellung gegenüber Wanderung.

Wenn die Pointe einer „postmigrantischen Perspektive“ (Yildiz 2019) darin liegt, MigrantInnen möglichst aus der Eigenperspektive sprechen und hören, forschen und handeln zu lassen, heißt dies an erster Stelle zu hören und zu sehen, was MigrantInnen „nach der Migration“ beschäftigt, in welche postmigrantischen Lebenswelten sie nach der Wanderung eingespannt sind, vor welchen postmigrantischen Aufgaben und Herausforderungen beim Niederlassen nach der Wanderung sie gestellt sind, welche Abdrücke ihre Schritte des Fußfassens in der „postmigrantischen Gesellschaft“ (Foroutan 2019) hinterlassen und wie sie diese Gesellschaft durch ihre Ankunft irreversibel verändern.

„Die empirische Tatsache“ anzuerkennen, „dass Migration nicht als Ausnahme von nationalen Vergesellschaftungsprozessen begriffen werden kann, sondern zentrale Normalität von Gesellschaft ist“ (Espahangizi et al. 2016: 15), ist schlichtweg zu wenig, um von Postmigration sprechen zu können. Die Faktizität und Normalität von Migration können - und dies geschieht bereits - auch Politiken, Diskurse und Institutionen einsehen und anerkennen, die den Umgang von Migration und MigrantInnen als Integration, Diversitätsmanagement oder Assimilation auffassen. Vielmehr liegt das distinktive Kennzeichen des „Postmigrantischen“ darin, die Faktizität und Normalität von migrantischem Ankommen geltend zu machen, darauf mit Nachdruck hinzuweisen, dass das, was sich in der postmigrantischen Gesellschaft abspielt, Ankommen ist. 
Es bietet sich auch aus empirischen Gründen an nach der Migration „nach der Migration“ zu suchen und das „Ankommen auf eigene Rechnung“ zum Fokus der postmigrantischen Bildgebung von Migration zu machen. Das Ankommen nach der Wanderung folgt nicht nur der Wanderung nach, sondern nutzt auch die Wanderung, kommt durch Wanderung zustande, kann Wanderung in sein Handlungsrepertoire einbeziehen und in sich Wanderung einschließen. So stellt sich das Ankommen „nach der Migration“ auch über die Wanderung als Prozess dar, der Wanderung umfasst und benutzt. Yildiz (2014) analysiert Arten und Strategien von Wanderung, die von AkteurInnen der zweiten oder dritten Generation genutzt werden, um „auf eigene Rechnung" anzukommen, um sich niederzulassen, sich von den Niederungen der Gesellschaft, an welche die Erste Generation Anschluss findet, abzuheben und sich Wege nach oben zu verschaffen. Anders als die Erste Generation haben solche postmigrantischen AkteurInnen keine direkten Erfahrungen mit Migration als (Aus- und Ein-)Wanderung gemacht (Bojadžijev/ Römhild 2014: 18). Solche PostmigrantInnen kennen hauptsächlich jenen Teil von Migration, der nach der Wanderung kommt, befassen sich mit Migration ausschließlich aus der Perspektive des Niederlassens, sind primär in Migration qua Niederlassen begriffen, sind vor Aufgaben des Niederlassens gestellt, die sie einfallsreich unter strategischer Einbeziehung von Wanderung mit Ähnlichkeit zu der bereits erwähnten Transmigration zu lösen versuchen.

In Hinblick auf die postmigrantischen Nachkommen kristallisiert sich noch eine wichtige Bedeutung von „post“ der Postmigration heraus. Für die im Ankunftsland geborenen und aufgewachsenen Nachfahren von MigrantInnen impliziert dieses „post“, rassismus- und herrschaftskritisch mit der fortdauernden Zuordnung in die Ecke des ,Migrantischen' Schluss $\mathrm{zu}$ machen, wo ihnen weiterhin symbolische Diffamierung gepaart mit soziostruktureller Deklassierung entgegenschlägt und die negative Aura als MigrantIn $z$ uelten mit vielfacher sozialer Benachteiligung von MigrantInnen verschränkt ist, nach dem Motto adding insult to injury. Das Aufgreifen von Postmigration als Thema und als Perspektive beflügelt das Ausbrechen aus der Ecke des ,Migrantischen', stützt und stärkt das Abwerfen negativ besetzter Semantiken des ,Migrantischen' sowie das Aufbegehren gegen die damit einhergehenden Benachteiligungen. Diese emanzipatorische und ermächtigende Wirkung des Perspektivenwechsels auf Postmigration gelingt an erster Stelle durch das
Hervorheben der Faktizität und Realität des Ankommens als positiver Kontrapunkt und Hauptteil des Migrationsphänomens.

Den Akzent auf Postmigration zu legen, heißt das "Ankommen auf eigene Rechnung" post oder nach der Wanderung und als Gegenpol wider die Wanderung zu betonen, es der negativen Aura des ,Migrantischen' als positives Gegenstück vorzuhalten und schließlich das Ankommen über die Wanderung und in die Pole-Position zu stellen. Diese Vorrangstellung hat zum einen damit zu tun, dass das Ankommen Wanderungsmobilität(en) einschließt und nicht umgekehrt. Zum zweiten schließt das Ankommen Wanderungsprozesse $\mathrm{ab}$, ist Grund und Ziel von Wanderungen. Menschen wandern aus, wandern durch, wandern ein, um anzukommen und um zu bleiben, und nicht umgekehrt. Dies macht wiederum auf ein drittes Moment aufmerksam, warum dem Niederlassen der Vorzug zu geben ist: Das Niederlassen ist ein wesentlich längerer Prozess als die davor liegende Wanderung. MigrantInnen sind länger, zeitlebens, oft auch generationsübergreifend mit dem Niederlassen beschäftigt.

Viertens bekräftigt die Vorrangstellung von Ankommen gegenüber Wanderung deren biografische und existenzielle Relevanz. Durch das Niederlassen wird die individuelle Lebensführung stärker und umfassender verändert als durch den spannendsten und abwechslungsreichsten Wanderungsverlauf.

Diese semantische Ausdifferenzierung des „post“ der Postmigration bringt verschiedene, ineinander verschachtelte Relationen zwischen Wanderung und Ankommen als Fokusse der Betrachtung von Migration zur Geltung. Das „post“ verweist nicht nur auf das zeitliche „nach“. Post-Migration ist das Ankommen von MigrantInnen auch in Bezug auf den Nexus der Migration, also in handlungspraktischer, und in weiterer Folge auch in biografischer, in existenzieller Hinsicht. In dem „post" der Postmigration zeigen sich verschiedene Dimensionen und Gründe der Überschreitung des Wanderungsmodells in Richtung Ankommen, die diese Kehrtwende (postmigration turn) der kritischen Migrationswissenschaft anstoßen.

\section{Als „MigrantIn“ gelten: laborieren am Ankommen} von unten

Die Fokusverlegung von Wanderung auf das Niederlassen wie insbesondere in den Forschungen zur Postmigration forciert, ist aber nicht nur auf kritische 
sozialwissenschaftliche Bildgebungen von Migration beschränkt. Alltagsdiskursive Adressierungen von ,Migranten', ,anders' und ,Andere' zu sein, nehmen nicht auf das Wandern Bezug, sondern betonen nachdrücklich das Ankommen. „Die Bezeichnung, Migrant_in“" visiert ”- paradoxerweise - in erster Linie nicht Wanderungserfahrung, sondern eher eine vermutete und zugeschriebene Abweichung von Normalitätsvorstellungen im Hinblick auf Biografie, Identität und Habitus" (do Mar Castro Varela/Mecheril 2011: 168) an. In niederschwelligen Diskursen des Alltags deutet als MigrantIn $z u$ gelten auf eine negativ-auratische ,Andersheit' hin, die sich nicht mehr aus Distinktionen sozialer Vielfalt (diversity) speist, welche neu und von außen herkommend, weitgehend geografisiert, ethnografisiert, kulturalisiert und vor allem egalitaristisch als gleichwertig und gleichstehend inszeniert werden. Die Andersheit der „Migrationsanderen“ (ebd.) ist negativ behaftet, sofern sie hierarchische Unterscheidungen von Über- und Unterordnung, von Über- und Unterlegenheit, von Oben und Unten bezeichnet und re-produziert. Diese Andersheit des ,Migrantischen ist eben anders als anders, verweist auf unten, dient als Wegweiser für das Ankommen von unten, ist nicht mehr a matter of diversity, sondern a matter of underclass, deutet nicht auf newcomers from abroad, sondern auf bottom comers from below hin.

Als MigrantIn gelten und als "Migrationsandere“ gehandelt zu werden, zeigt auf unten und auf das Ankommen, auf das Ankommen unten, was die migrantische Art anzukommen ist - nicht von außen, wie es gemeinhin angenommen wird und geografisch betrachtet auch stimmt, sondern von unten. Es handelt sich hier um eine doppelte Weiterleitung von anders auf unten und von unten auf das Ankommen.

Jene, die als „Migrationsandere“ gelten, kommen von unten an und gelten als „Abweichung von Normalitätsvorstellungen in Hinblick auf Biografie, Identität und Habitus“ aus ein und demselben Grund. Die minimale Schwelle gesellschaftlicher Normalität im Kontext des gegenwärtigen Liberalismus und Kapitalismus liegt in der Ankoppelung an deren Spielwiesen und Spielweisen. Ist man von diesen abgekoppelt - wie jene, die als „Migrationsandere“ gelten, per Herkunft und infolge der postkolonialen Drift der kapitalistischen Welttektonik abgekoppelt sind -, fällt man von der gesellschaftlichen Normalität ab und kann beim Versuch, Anschluss zu finden, dies nicht anders als von unten schaffen und nirgendwo anders als unten ankommen. Von unten angekommen zu sein, bedeutet aber nur, die elemen- tare Bedingung gesellschaftlicher Normalität erfüllt zu haben und daher noch lange und in vielerlei Hinsicht als „Abweichung von Normalitätsvorstellungen“ gehandelt zu werden, gerade weil man als MigrantIn knapp an der minimalsten Normalitätsschwelle Fuß fasst und auf Dauer, oft über Generationen hinweg, mit der realen Möglichkeit konfrontiert ist, unter diese abzurutschen und ein Leben an der Schwelle zu fristen.

Vor dem Hintergrund besagter Adressierungen von MigrantInnen als „Migrationsandere“, als Neuund Unten-Angekommene, fällt kontrastreich auf, dass durch den in politischen und wissenschaftlichen Diskursen geläufigen Begriff ,MigrantIn` eine kategoriale Vermengung von realen Gegensätzen vollzogen wird. Es werden unter dasselbe kategoriale Dach sowohl jene gesteckt, die als MigrantInnen gelten, sofern sie mehr oder weniger von den Spielwiesen und Spielweisen der liberal-kapitalistischen Welt abgekoppelt sind, als auch die privilegierten Eliten (wie etwa ManagerInnen, AkademikerInnen, Schlüsselkräfte), die vom liberalkapitalistischen Spielgeschehen am meisten profitieren und durch ihre Hypermobilität oft in sachlicher Hinsicht MigrantInnen sind (aufgrund von Herkunft oder Staatsbürgerschaft), ohne aber als solche zu gelten.

Es fällt gerade dann stärker auf, dass Menschen als MigrantInnen gelten, wenn diese nach sachlichen Kriterien wie Staatsbürgerschaft oder Herkunft keine MigrantInnen mehr sind, weil bereits StaatsbürgerInnen des Ankunftslandes, weil bereits dort geboren und aufgewachsen. Umgekehrt gelten oft nicht als MigrantInnen viele jener, die nach den Sachkriterien von Staatsbürgerschaft (weil fremde StaatsbürgerInnen) oder Herkunft (weil im Ausland geboren und aufgewachsen) oder Wanderung (weil unaufhörlich in Wanderung begriffen) MigrantInnen sind, was auf ManagerInnen, Schlüsselkräfte, akademisches Personal, Fachkräfte, InvestorInnen, also auf all jene zutrifft, die bestens in die liberal-kapitalistische Weltszenerie eingebunden sind.

So eine kategoriale Vermengung unterbindet die kritische Auseinandersetzung zwischen und mit den Gegensätzen. Die kritische Feststellung, dass MigrantInnen von unten ankommen, ist schwer möglich, wenn der Dachbegriff ,MigrantIn' auch jene umfasst, die sich oben bewegen, und wenn und wo sie ankommen, dies jedenfalls nicht von unten tun. Die kritische Einsicht, dass migrantische Anschlussfindung von unten erfolgt, kann dann als unzulässige Verallgemeinerung zurückgewiesen werden. Einzig unzulässig hier ist aber die kategoriale Vermengung der realen 
Gegensätze. Ob Zufall oder Strategie, verschweigt so eine kategoriale Vermengung den realen Gegensatz und bringt vor allem jene zum Schweigen, die dabei im Nachteil liegen. Die einzige Möglichkeit, die Stimme der Kritik nicht verstummen zu lassen, dass Menschen als „Migrationsandere“ gelten, sofern sie von unten herkommen und unten ankommen und lang an den Schwellen gesellschaftlicher Normalität laborieren, liegt darin, die besagte kategoriale Vermengung realer Gegensätze zu entwirren und die Unterscheidung zwischen jenen, die als MigrantInnen gelten (auch wenn sie keine mehr sind), und jenen, die MigrantInnen sind (und trotzdem nicht als solche gelten), klar zu ziehen - eine Unterscheidung, die Kritik bedeutet und Kritik ermöglicht.

Durch den Perspektivenwechsel von Wanderung auf das Ankommen, der hier anhand von verschiedenen Pfaden, in kritischen (wissenschaftlichen, theoretischen) Bildgebungen von Migration wie auch in realistischen (nicht immer kritischen und nicht unbedingt theoretischen) Adressierungen von „Migrationsanderen“ oder jenen, die als MigrantInnen gelten, rekonstruiert werden konnte, wird die Bahn auf die full vision auf Migration frei gemacht.

\section{Die full vision: der Nexus von Migration}

In full vision zeigt sich uns der Nexus von Wandern und Ankommen nicht nur als a matter of migration, sondern vor allen Dingen als a matter of capitalism. ${ }^{13}$ Er verbindet das, was der Kapitalismus trennt. Dabei steuert das Ankommen, nicht das Wandern, bei diesem Verbinden das Binden bei.

Der Nexus der Migration ist im „Weltsystem“ des Kapitalismus (Wallerstein 2019) eine zentrale tektonische Struktur, ja sogar ein Prinzip der Gestaltung dieser Weltszenerie von ihren historischen Anfängen bis in die Gegenwart. ${ }^{14}$ Die Weltsystemtheorie Immanue Wallersteins ortet eine ungleichordnende Dynamik der postkolonialen Drift, d. h. des Auseinanderdriftens und Untereinanderdriftens von Standorten, welche die

13 Zum Zusammenhang von Migration und Kapitalismus siehe Sassen 2017.

14 „Weltsystem“ ist der Kapitalismus, sofern er flächendeckend über den Globus waltet. Die Rede vom „Weltsystem" ist auch dadurch berechtigt, dass der Kapitalismus nicht nur eine Art zu produzieren, $\mathrm{zu}$ arbeiten, $\mathrm{zu}$ wirtschaften ist, sondern alle Lebensbereiche infiltriert und alles durchdringt, was man in der Welt macht, was die Welt ausmacht und sie zur Welt macht.
Tektonik des kapitalistischen Weltsystems strukturiert und hierarchisiert.

Die ungleichordnende Dynamik des Auseinanderdriftens von Positionen und Lokationen im kapitalistischen Wertschöpfungsprozess ist dem Kapitalismus in die Wiege gelegt als wirtschaftliches System, welches durch den „Imperativ der unendlichen Akkumulation von Kapital“ (Wallerstein 2019) AkteurInnen der Wertschöpfung zu Wachstum, Innovation und Expansion zwingt und zur Selbststeigerung antreibt. Insbesondere wird die Dynamik der postkolonialen Drift durch den Imperativ zur Expansion vorangetrieben.

Im Gleichschritt mit der kapitalistischen Ökonomie zeichnet sich die postkoloniale Drift auch im Feld der Politik und anhand von Indikatoren des politischen Liberalismus ab. Für dieses Zweiergespann von Kapitalismus und Liberalismus sind Embleme wie „liberaler Kapitalismus" deshalb nicht passend, weil so der in Wirklichkeit vielseitige Nexus von Kapitalismus und Liberalismus mit dem laissez-faire oder „liberalen“ Kapitalismus des 19. Jahrhunderts (Fraser 2019: 87ff.) verwechselt werden kann. Der Nexus von Kapitalismus-Liberalismus ist breiter gefasst und umfasst mehr als der Kapitalismus als ein historisches, ökonomisches oder gesellschaftliches Phänomen. Im besagten Nexus spielt auch der bunte Komplex theoretischer, ideologischer, institutioneller und politischer Hebel der Liberalisierung, begriffen als Prozess von Freiheitszunahme und Beseitigung allfälliger Hindernisse auf dem Weg kapitalistischer Steigerungsdynamiken, eine ebenso wichtige Rolle. Die gesamte Bandbreite des Kapitalismus-Liberalismus-Nexus der Moderne vervollständigen Demokratie und Wohlfahrtsstaat als die historisch gewachsenen Gegengewichte der Steigerungsdynamik kapitalistischer Wertschöpfung und politischen Liberalismus. An den Top-Adressen im Nexus von Kapitalismus-Liberalismus haben sich Demokratie und Wohlfahrtsstaat als jene Institutionen herausgebildet und dort Top-Errungenschaften erbracht, die exzessive Entwicklungen von Kapitalismus-Liberalismus abfangen und im Zaum halten können. Es driften tektonische Elemente auseinander und untereinander, die nie nur ökonomische Standorte des Kapitalismus sind, sondern immer auch mit verschiedenen empirischen Ausformungen des politischen Liberalismus, liberaler Demokratie, Rechtsstaatlichkeit, Wohlfahrtstaatlichkeit usw. gekoppelt oder von diesen teilweise oder komplett abgekoppelt sind. In seinem vollen Ausmaß erwirkt die postkoloniale Drift nicht nur die Abkoppelung von den gains ökonomischer Steigerung und 
politischer Liberalisierung, sondern auch die Abkoppelung von demokratischen und sozialstaatlichen TopErrungenschaften, welche den pains, den Exzessen und Krisen ökonomischer Steigerung und politischer Liberalisierung entgegensteuern und diese eindämmen können.

Die Drift bestimmt und wird daran gemessen, ob die jeweiligen Arenen mehr von den Vorteilen oder mehr von den Nachteilen kapitalistischen Wachstums, technologischer Innovation, von Mobilität und Liberalisierung zu bieten haben und auch ob und mit welchen Freiheiten und Rechten, mit welchen Chancen zur Bildung, Gesundheitsversorgung und vielem mehr man auf den jeweiligen Arenen rechnen kann. TopAdressen von Kapitalismus und Liberalismus stellen solche Spitzenerrungenschaften griffbereit und ermöglichen den Zugriff darauf, anders als die weiter unten angesiedelten Arenen, wo sich gains and pains made by capitalism and liberalism in umgekehrt proportionalem Verhältnis gegenüberstehen und das Abnehmen von gains in das Zunehmen von pains übergeht. Bildgebungen von globaler Ungleichheit, von der ungleichen Verteilung und Konzentration vom Wohlstand (z. B. World Bank 2017; Milanovic 2016) oder auch von „uneven geographical developments“ (Harvey 2005: 87 ff.) indizieren die Stärke der systemtektonischen Drift und die Tiefe der Klüfte, die sie aufreißt. Migrationen hingegen liefern ihrerseits Indizien (z. B. IOM 2019b) über die Richtung des Driftens und auch darüber, dass es sich um ein Auseinanderdriften handelt.

Wallersteins Weltsystemtheorie kartiert die kapitalistische Welttektonik in hierarchisch unter- und übergeordnete tektonische Elemente von „Zentrum“, „Semiperipherie“ und „Peripherie“. Würde man die Effekte der Ungleich- und Schlechterstellung der postkolonialen Driftdynamik mehr hervorheben wollen, lassen sich in der Tektonik des weltumspannenden Nexus von Kapitalismus und Liberalismus Top-, Bottom- und Non-Spots ausfindig machen. Die verschiedenen ungleichstehenden und ungleichstellenden Lokationen werden danach definiert, ob und wie sehr sie von den Spitzenerrungenschaften und -angeboten sozialer, politischer und ökonomischer Art an- oder abgekoppelt sind, die gegenwärtig im globalen Zusammenhang von Kapitalismus und Liberalismus am selben Ort und zur selben Zeit verfügbar sind.

Der Haupteffekt dieser Drift-Dynamik ist die postkoloniale Abkoppelung von Herkunftsorten in und von diesem liberal-kapitalistischen Weltsystem, mit welcher die Menschheit nach dem Zerfall der kolonialen Welt- tektonik zu leben hat. Die postkoloniale Abkoppelung distanziert (horizontales Driften) und hierarchisiert (vertikales Driften) ungleiche und ungleichstellende Standorte im gegenwärtigen Weltsystem. Dadurch wird in bisher ungeahnten Ausmaßen und mit unüberschaubaren Konsequenzen Ungleichheit aufgrund von Herkunft (Milanovic 2016) hergestellt und es werden durch eine Art „Birthright-Lottery“ (Shachar 2009: 4) oder Herkunftsort-Lotterie Besser- und Schlechterstellungen verteilt, die sich auf Menschen in big numbers und auf gesamte Herkunftswelten auswirken.

Den Grenzfall postkolonialer Abkoppelung stellen Herkunftsarenen dar, die jenseits der Außenränder des liberal-kapitalistischen Weltsystems abgehängt sind und als Non-Spots durch die „International

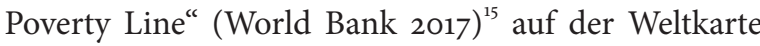
eingetragen sind. Solche komplett abgedrifteten BlindSpots markieren das anwachsende Außen der liberalkapitalistischen Weltszenerie, sind aber empirisch und quantitativ gar nicht so insignifikant und unsichtbar, fallen geografisch bspw. mit großen Kontinentalteilen (wie Subsahara-Afrika) zusammen, belegen große Territorien und beherbergen erhebliche Anteile der Weltbevölkerung.

Ist die liberal-kapitalistische Weltszenerie gegenwärtig tektonisch in Top-, Flop- und Non-Spots unterteilt, mit dazwischen aufklaffenden, größer und unpassierbar werdenden Klüften der postkolonialen Drift, bringt sich der Nexus der Migration in dieser Systemtektonik und -dynamik mit dem Haupteffekt ein, in struktureller Hinsicht die Klüfte zur überbrücken, to close the gaps zwischen best and the rest. ${ }^{16}$ Der Nexus von Wandern und Niederlassen ist auch dynamischer Widersacher der gesamttektonischen Drift. In ihm entfaltet sich insofern die Dynamik von bottom hoppen jener, die als MigrantInnen gelten, wie diese, von den Top-Arenen relativ oder absolut abgehängt, sich den Anschluss daran aktiv, durch Wandern und Niederlas-

15 Von derzeit 1,90 Dollar pro Person und Tag.

16 Was Begehren nach einem „neuen Kosmopolitismus von unten“ anfeuert (Römhild 2009: 234). Tatsächlich stellen Migrationen Verbindungen her, die den Kosmopolis näherrücken lassen, tatsächlich arbeitet der Nexus der Migration auf den Kosmopolis hin, den umgekehrt gerade die postkoloniale Drift der liberal-kapitalistischen Welttektonik systematisch verhindert. Die reale Unmöglichkeit der Welt als Gemeinwesen, als Kosmopolis unter den tektonischen und dynamischen Begebenheiten des liberal-kapitalistischen Weltsystems ist ein weiterer Grund und eigentlich der Hauptgrund, hier von einer postkolonialen Abkoppelung per Herkunft und nicht bloß von Exklusion auszugehen. 
sen, erarbeiten und erkämpfen. Das ,Weghüpfen ` von Herkunftsorten und Herkunftsarten, die ,am Boden sind oder den Boden der Welttektonik von Kapitalismus und Liberalismus bilden, und das Hinaufhüpfen am bottom, an den unteren Enden der Top-Spots, ist das, was man wortwörtlich als bottom hoppen bezeichnen kann. MigrantIn hüpft vom (schlechter gestellten) bottom zum (besserstellenden) bottom ab und hinauf, erzielt dadurch auch sozialen Aufstieg, den paradoxen Aufstieg nach unten.

Zum Wechselverhältnis zwischen Driftdynamik und Migrationsnexus gehört in systemdynamischer Hinsicht aber mehr als das Entgegenwirken und Entgegenlaufen der postkolonialen Drift. Nicht nur entsteht Migration als Nexus von Wandern und Niederlassen in Reaktion auf das postkoloniale Aus- und Untereinanderdriften der liberal-kapitalistischen Gesamttektonik, sondern wird umgekehrt auch die Driftdynamik erheblich durch Migration enerviert und aktiviert. Driftbewegungen von tektonischen Platten bleiben über längere Zeiträume unbemerkt, und wie die Klüfte, die sie aufreißen, harren Driftbewegungen in einem durchaus latenten, inaktiven Zustand aus, was sich schnell ändert, sobald MigrantInnen am Horizont erscheinen. Dann materialisieren sich Drift und Klüfte schlagartig, nehmen verschiedene entwicklungspolitische, grenzpolitische, migrationspolitische, integrationspolitische, national- und supranationalpolitische Formen an. Durch Migrationen angereizt, verdichtet sich die postkoloniale Drift heutzutage in der tektonischen Konstellation der ,Festung Europa' Auch die Kluft zwischen best und rest materialisiert sich in den Meerestiefen des Mittelmeeres.

Die Kritik am Wanderungsmodell der Migration und die Fokusverlegung auf das Ankommen gipfeln in dieser full vision auf Migration als Nexus von Wandern und Ankommen und werfen auch in Hinblick auf die bereits angeschnittenen wissenschaftlichen und außerwissenschaftlichen Bildgebungen von Migration als Ankommen die Frage auf: Was ist dann das Ankommen? Worum geht es überhaupt beim Niederlassen von MigrantInnen?

\section{Den Dispositiven zuwider}

Ließe man, der postmigrantischen Theorie folgend, MigrantInnen bezeugen und bestimmen, was Niederlassen ist, würde das Ankommen zuallererst mit dem Einrichten eines neuen Zuhauses, mit dem Fußfassen auf einem neuen Boden, mit dem Aufbau einer neuer
Existenz, mit Prozessen, Schritten, Episoden und Aufgaben dessen assoziiert, was man fachlich auch "homing“ (ein Zuhause errichten) nennt. Doch wird es selten den MigrantInnen überlassen, das Ankommen in Eigenregie und „auf eigene Rechnung“ (Yıldız 2014: 24 ff.) durchzuführen. Beim Niederlassen werden MigrantInnen nie in Ruhe gelassen, dort, dann und auf ihre Weise Fuß zu fassen, sondern sie werden andauernd in Unruhe versetzt, in Ungewissheit und Unsicherheit über Ort, Gründe, Dauer, Rechte und Aussichten des Niederlassens gehalten.

Aufnahmestaaten haben ein strategisches Interesse, das Ankommen von newcomers zu dirigieren, und ebenso fixe Vorstellungen über den Ankommensablauf und über die Rollen, die MigrantInnen im aufnahmestaatlich abgesegneten Niederlassensszenario zugeteilt oder verwehrt werden, unabhängig davon, ob dies dem Zuhausefinden/Zuhausesein („homing“) von MigrantInnen entspricht oder widerspricht, ob so Fußfassen ermöglicht oder eher erschwert wird.

Aufnahmestaaten dirigieren newcomers und bottom comers beim Fußfassen sozusagen in den ,falschen Film ' vom Niederlassen hinein, der entweder ,Integration' oder ,Diversitätsmanagement' oder beides heißt. Hier schleicht sich die Idealisierung von Migration als Wanderung durch die Hintertür herein, verstrickt in den gängigen Konzeptualisierungen vom Ankommenssprozess als Integration oder Diversitätsmanagement. Das Ankommen wird in beiden Fällen durch das Prisma der Wanderung betrachtet.

Integration zu Aufgabe und Ziel der Gesamtgesellschaft sowie als staatspolitisches Dispositiv ${ }^{17}$ zu machen, stellt das Ankommen von MigrantInnen als ein gigantisches Brems- und Anhaltemanöver gegenüber Wanderung, und im Speziellen gegenüber der ausklingenden Ein-Wanderung dar. Im Integrationsmodell wird die Aufnahmegesellschaft als ein solider Be-hälter postuliert mit entsprechendem Haltevolumen und Grenzen logistischer Aus-haltbarkeit von Migration, in dem alles, was mit ausklingender Ein-wanderung getan und

17 Warum Nationalstaaten es nicht lassen können, Menschen und insbesondere MigrantInnen zu integrieren, und warum für sie Integration indisponibel ist, siehe Bojadžijev 2018. Das Besondere des Dispositivs als Handlungsart ist es, eine bestimmte Vorgehensweise nicht sein lassen zu können, welche sehr wohl unterlassen oder auf eine alternative Weise hätte ausgeführt werden können: Integration muss nicht sein, obwohl sie alternativlos erscheint. Migrantisches Ankommen kann sehr wohl ohne Integration auskommen. 
den Ein-wandernden angetan wird, dem obersten Ziel des An-Haltens untergeordnet ist. Zu den bekanntesten praktischen Anwandlungen der Integrationslogik des Großen Haltens gehören unter anderem: Newcomers an der Grenze an-halten und dabei kontrollieren ihnen Auf-Enthalt geben und dabei kontrollieren, zur Sicherung vom Lebens-unterhalt verpflichten und dabei kontrollieren, Ab-Haltung von Sprachprüfungen verordnen und dabei kontrollieren, Ein-Haltung von aufnahmegesellschaftlichen Werten und Hausregeln, Gesetzen und Fristen abverlangen und dabei kontrollieren. Alle diese Zwischenstationen des Bremswegs zur völligen Anhaltung von Einwanderung steuern auf den Wunschzustand hin, Zusammen-halt in der Gesellschaft zu ermöglichen und den Integrationsweg durch die Staatsbürgerschaftsübernahme abzuschließen.

Spielt das (An-)Halten von Ein-wanderung beim Auffassen von Ankommen als Integration groß auf, verbirgt auch das Emblem der ,Diversität' eine ähnlich auf Wanderung zentrierte, aber dennoch davon abweichende Sicht- und Herangehensweise auf Ankommen. Ankommen unter Diversität zu subsumieren und als Prozess des Managements von Diversität zu begreifen, heißt zum einen den Prozess des Niederlassens weiterhin mit Fokus auf Wanderung zu thematisieren, dies aber, anders als das Integrationsnarrativ, von der Seite der Aus-Wanderung, des Her-Kommens anzufassen und die Politik der Diversität auf das Akkommodieren der Vielfalt der Herkünfte auszurichten. In Anlehnung an das vorhin angesprochene Konzept des ,Integrationsdispositivs' kann man auch die diversitätspolitischen Programme und Praktiken als ,Diversitätsdispositiv' bezeichnen. ${ }^{18}$ Es sind meistens die unterhalb der staatlichen Ebene angesiedelten kommunalen Verwaltungen, die es nicht lassen können, migrantisches Ankommen als Akkommodieren der Diversität der Herkünfte zu gestalten, die migrationsbezogene Vielfalt $\mathrm{zu}$ administrieren (durch eigens dafür geschaffene Magistratsabteilungen), zu studieren (durch Diversitätsmonitore), zu zelebrieren (durch Diversity-Balls), die lokale Öffentlichkeit über Vielfalt aufheiternd zu informieren mit dem Zweck, von Vielfalt zu profitieren, was Diversitätsmanagement auf eine ähnliche Weise auf lokaler Ebene zum Dispositiv macht, wie die Integration für Nationalstaaten zum Dispositiv wird.

Während im Integrationsszenario die Einsicht, dass es sich beim Ankommen um das Ankommen, um

18 Zum historischen Werdegang von Diversitätsmanagement siehe Kelly/Dobbin 2001. zu bleiben und nicht um eine Wanderung handelt, an das Ziel verloren geht, ausklingende Ein-Wanderung mit allen Mitteln und auf verschiedene Weisen anzuhalten, wird in dem Diversitätsszenario der Blick auf das Ankommen durch die übergeordnete Aufgabe verstellt, mit dem endlos prolongierten Herkommen verschiedenartiger Menschen in big numbers zurechtzukommen, die Vielfalt ihrer Herkünfte zu managen, indem man Vielfalt administriert, exploriert, zelebriert, darüber informiert, davon profitiert. Auf diese Weise verschreibt man sich nicht nur dem Ziel vom gesellschaftlichen Zusammenhalt an, wie es dem Integrationsdispositiv vorschwebt, sondern steuert das Szenario der Diversität auf das anspruchsvollere Ziel von Zusammenleben der verschiedenartigen Vielen hin.

\section{Migrantisches Ankommen ist Ankommen von unten}

Migrantisches Ankommen nicht reflexartig in die Deutungsklammern von ,Integration' oder ,Diversitätsmanagement' einzupassen, bedarf einer Fokusänderung. Ähnlich wie im kritischen Konzept von Migration setzt auch die kritische Bildgebung von migrantischem Ankommen damit an, den Fokus auf Wanderung aufzuheben, der den gängigen Idealisierungen des Niederlassungsprozesses als Integration oder Akkommodation von Vielfalt zugrunde liegt. Anstatt auf Wanderung wird der Fokus auf das Ankommen selbst als Akt und Prozess vom Fußfassen gesetzt.

Diese Fokusverlegung impliziert, Ankommen nicht nur zum Erkenntnisgegenstand zu haben, sondern es zum Standpunkt zu machen und in weiterer Folge ihm auch als Erkenntnismethode zu folgen. Diese methodologische Wendung und Verwendung des migrantischen Ankommens misst dem Fußfassen eine methodische Rolle bei. ${ }^{19}$ Das Fußfassen ist das Schlüsselglied der Wirkungskette des migrantischen Ankommens. Es methodologisch zur Richtschnur der Erkenntnisgewinnung zu machen und es entlang dieser Richtschnur kritisch zu analysieren, heißt dem Prozessverlauf und der Komposition vom Fußfassen besondere Achtsamkeit zu schenken, das Fußfassen in seine Komponenten auseinanderzunehmen und daraus die Leitfäden der kritischen Bildgebung vom Niederlas-

19 Eine vergleichbare Wendung und Verwendung eines Erkenntnisthemas als Erkenntnismethode legt der „border as a method“-Ansatz vor (Mezzadra/Neilson 2013: 14ff.). 
sen zu entwickeln. Die methodologische Devise lautet: Fußfassen eruieren, indem man es auch praktiziert, als jemand exploriert, der/die selbst im Fußfassen begriffen ist, und dabei notiert, was ihm/ihr dabei begegnet, was dabei auffällt, was mit ihm/ihr (also nicht nur mit den ,Anderen') passiert in den vielen miteinander verketteten Episoden und Sequenzen des Fußfassens.

Das Achten auf den Prozessverlauf und die Komposition vom Fußfassen als jemand, die und der selbst mittendrin im Fußfassen ist, geht auch mit einer weiteren, nunmehr epistemologischen Umstellung, mit der Veränderung der Art der gewonnenen Erkenntnisse einher. Das Ankommen als Methode bringt die Erkenntnis weg von dem Pfad der auf mathematischer Exaktheit ausgerichteten sozialwissenschaftlichen Erkenntnis. Durch das Fußfassen als Richtschnur des Erkenntnisprozesses wird vielmehr auf eine ganz andere Epistemologie der Spurensuche und Spurenlese (Ginzburg 1995) gesetzt.

Diese Umstellung hat zuerst und unmittelbar damit zu tun, dass das Fußfassen als Handlungsart und die Spurensuche und Spurenlese engstens miteinander verschränkt sind. Das Fußfassen hinterlässt Fußabdrücke und sucht, verfolgt und nutzt bereits bestehende Fußabdrücke, tritt sozusagen in die Fußstapfen der VorgängerInnen, orientiert sich an den bereits gebahnten Pfaden im Terrain. Umgekehrt ist auch die Erkenntnis über und von Spuren am schärfsten, wenn man selbst in eine Aktivität des Fußfassens involviert ist, welche selbst Pfade spurt.

Ankommen als Fokus und Methode in einem auf Spurensuche und Spurenlese ausgerichteten Erkenntnismodell anzuwenden, prägt auf entscheidende Weise auch die kritische Bildgebung von migrantischem Ankommen, die sich den vorherrschenden Narrativen und Dispositiven von Integration und des Managements von Diversität entgegenstellt. Zum einen ist die Fokuslegung auf das Ankommen ein Schritt der critique from below (Kritik von unten). Dass es sich dabei um critique from below handelt, liegt daran, dass migrantisches Ankommen, wie wir bereits gesehen haben, nirgendwo anders als unten ansetzen kann und sich auf Dauer unten abspielt.

Die zweite Konsequenz folgt aus der ersten und hat mit der Machart von Kritik from below zu tun. Dafür ist in unserem Kontext vor allem einer der bekanntesten Vorstöße der critique from below erhellend: das programmatische Vorhaben der Indian Subaltern Studies Europa zu ,provinzialisieren' (Chakrabarty 1992, 2000), die epistemische Dominanz, die Zentralität europäi- scher Perspektiven, Themen und Erkenntnisarten über best and the rest, also über Europa als best und alles Nicht-Europäische als rest, abzusetzen und umzukehren.

Der programmatische Griff der ,Provinzialisierung' der Zentralinstanz der Erkenntnis - ob dies wie in historischen Forschungen oder in Theorien der Moderne, der Blick auf und von Europa oder im vorliegenden Kontext das Wanderungsparadigma der Migration ist - zielt nicht bloß auf den Platztausch von Zentrum und Rand, zentralen und marginalen Perspektiven ab. Vielmehr entwirft der Wurf der ,Provinzialisierung eine „history that does not yet exist“ (Chakrabarty 1992: 20), stellt ein ,Indien“ in den Fokus der Geschichtsschreibung, das nicht bloß als das ,Andere-von-Europa' erzählt wird oder sich erzählt.

Die Kritik am Wanderungsmodell der Migration, welches, wie an den Konzepten von Integration und Diversitätsmanagement ersichtlich, auch die Szenarien von migrantischem Niederlassen vorschreibt, wendet dieselbe Wurftechnik der ,Provinzialisierung' an, bringt ein Ankommen ins Zentrum der Bildgebung auf Ankommen, das Wanderung als Fokus nicht nur ersetzt, sondern sich von Wanderung als Fokus komplett absetzt. Das ist genau das, worauf das Ankommen als Fokus und Methode der Forschung hinwirkt, nämlich Ankommen zu verstehen und zu untersuchen, ohne nach Wanderung Ausschau zu halten und ohne es in Bezug auf Wanderung, auch nicht als das Anderevon-Wanderung, zu definieren. Man bleibt auf den Prozess des migrantischen Ankommens, auf den Ablauf des Fußfassens, auf das System von Fuß und Boden sowie auf deren points of contact und dynamische Verhältnisse fokussiert und muss dabei als Allererstes erkennen, dass das migrantische Niederlassen nicht bloß irgendein Fußfassen ist, sondern ein nieder-Fußfassen-lassen, ein Ankommen, das ungleich und unten stellt, bei dem Ungleichheit und das Unten auffallend sind.

Migrantisches Ankommen mit Fokus auf das Ankommen selbst, durch das Ankommen als ,Methode mit erhöhter Achtsamkeit auf den Prozessverlauf und die Komposition vom Fußfassen zu untersuchen, hat eine Reihe von Implikationen nicht nur für die (kritische und spurensuchende) Art der Erkenntnis, die dadurch gewonnen wird. Ebenso wichtig ist der inhaltliche, thematische Ertrag dieser Umpolung des Ausblicks auf Ankommen, weg von der allgegenwärtigen Wanderung, hin zum Ankommen abseits und nach der Wanderung („Nach der Migration“ qua Wanderung, wie die Devise der Postmigrationstheorie lautet). 
Die Fokussetzung auf das Fußfassen setzt uns in der Untersuchung von migrantischem Ankommen sozusagen ein Ungleichheitsvisier auf. Vor allem wird die Aufmerksamkeit auf die Beschaffenheit und insbesondere auf die Unebenheiten des Terrains sowie auf the points of contact, auf die kritischen Nahtstellen und die Reibungsflächen, geschärft, die sich beim Fußfassen zwischen Fuß und Boden ergeben und somit neue Schritte und das Fortschreiten selbst überhaupt erst ermöglichen. Für die kritische Bildgebung vom Ankommen impliziert dies besondere Acht auf Ungleichheiten, Hindernisse, Schlechterstellungen, Ausrutscher, aber auch auf Widersprüche und Polarisationen zu geben, die sich beim Niederlassen von Menschen in big numbers ergeben.

Hierzu stellen empirische Forschungen zum migrantischen Niederlassen weiterführende Spuren bereit. Empirische Studien der OECD (2018) und OECD/ EU (2018) spiegeln die Trajektorie von migrantischem Ankommen wider. Die allerersten und allerwichtigsten Andockstationen für Neuankommende sind dabei die Großstädte, der Arbeitsmarkt und der Wohnmarkt und überall fassen Neuangekommene in diesen Bereichen unten Fuß (OECD 2018). Lassen sich MigrantInnen und im Speziellen Neuangekommene territorial konzentriert in urbanen Räumen nieder, steigt dort dadurch nicht nur der Bevölkerungsanteil von Menschen mit Migrationshintergrund insgesamt an, sondern wächst im Vergleich dazu überproportional auch der Anteil von Menschen mit Migrationshintergrund in den unteren und untersten Sparten von Arbeit und Bereichen von Wohnen, wortwörtlich in den unteren Etagen und qualitativ schlechteren Segmenten des großstädtischen Wohnbestands sowie in den Niedriglohnsektoren. ${ }^{20}$ Besonders deutlich zeigt sich das Ankommen von unten bei Geflüchteten (Huke 2020) und bei Neuangekommenen (der ersten Generation, Gans 2009), wirkt aber auch über die erste Generation hinaus weiter (Papademetriou et al. 2009). Neuangekommene schneiden entlang aller „Indicators of Immigrant Integration" (OECD/EU 2018) in Relation zu vergleichbaren alteingesessenen Gruppen schlechter ab. Ihr Neuankommen präsentiert sich als Ankommen von unten. Es erhöht den Anteil von MigrantInnen an

20 Ähnliche Schlüsse legen die Befunde zum Abschneiden von Neuankommenden in Feldern wie Bildung, Übergang von Schule zum Arbeitsmarkt, Arbeitssuche, Arbeitslosigkeit, Armut, soziale Inklusion, Gesundheit nahe (OECD/EU 2018). der Gesamtbevölkerung, aber noch mehr steigt durch das Ankommen der Anteil von MigrantInnen an dem gesellschaftlichen Bodenpersonal. ${ }^{21}$

Durch die Einblendung der Ungleich- und Schlechterstellungen, der Abrutscher nach unten, die den Menschen in big numbers beim Ankommen passieren, präsentiert sich das migrantische Ankommen, wenn mit Fokus auf das Fußfassen nicht auf Wanderung betrachtet, vor allem als Ankommen von unten. Auf diese Weise können kritisch gängige Vereinfachungen und Übertreibungen umgeworfen werden, die sich migrantisches Ankommen als Arrivieren, Koffer auspacken, Sesshaftwerden ausmalen oder dieses Ankommen in den Bilderrahmen des Neuankommens stecken, MigrantInnen über Generationen hinweg mit der ewigen Jugend beglücken, für immer neu zu sein, oder die dieses Ankommen von ,Fremden', ,Ausländern 'als Ankommen von außen auffassen. Von hier aus können auch die Polarisationen und Widersprüche weiterverfolgt werden, die auf Realitäten der Ungleichheit und Schlechterstellung des migrantischen Ankommens zurückgehen und sich an diversen points of contact im Prozess des Niederlassens abzeichnen. ${ }^{22}$

9. Migrationsgesellschaft? Die Arena, wo Menschen in big numbers von unten und in Widerspruch ankommen

Die Polarisation des Ankommens setzt bereits mit der postkolonialen Abkoppelung von Herkunftsorten von den Top-Adressen und Top-Angeboten im „Weltsystem“ des gegenwärtigen Kapitalismus an. Als Abgehängte von den Top-Adressen der gegenwärtigen liberal-kapitalistischen Weltszenerie können jene, die als MigrantInnen gelten, den Anschluss zunächst von unten suchen und finden. Vom ersten Schritt an ist das migrantische Ankommen mit der Ambivalenz behaftet, ein Niederlassen zu sein, das zugleich ein

21 ,Bodenpersonal' bezeichnet all jene Jobs an einem Flughafen, die stationär sind und die logistische Basis bereitstellen, um anderen (den Passagieren) zu ermöglichen, mobil oder hypermobil zu sein. Hier sind metaphorisch all jene gemeint, die in den verschiedenen Ungleichheitsskalen und Hierarchieordnungen der Gesellschaft unten rangieren, erhebliche Teile der Restbevölkerung auf ihren Schultern tragen und ihnen eine privilegierte oder schlichtweg, normale‘ Lebensführung ermöglichen.

22 Naika Foroutans Analyse der „postmigrantischen Gesellschaft“ (2019) zeichnet eine Reihe an Ambivalenzen, Widersprüchen, Polarisationen auf, die Deutschland als Ankunftsgesellschaft charakterisieren. 
nieder-Fußfassen-lassen, also Anschlussfindung an den untersten Ebenen der Gesellschaft ist.

Beim Ankommen an den untersten Sprossen der sozialen Leiter Fuß zu fassen, spült Neu- und UntenAngekommene, aber auch jene, die schon länger am Ankommen laborieren, in die Fänge spätkapitalistischer Ausbeutung. Hier zeichnen sich weitere Widersprüche ab. ,Spätkapitalistisch 'sind die Realitäten und Verhältnisse von Ausbeutung, die anders als ,kapitalistische Ausbeutung' (Wright 2005) nicht mehr hauptsächlich auf Arbeit bezogen, sondern auf den gesamten Lebensprozess entgrenzt sind, die nicht primär Arbeitskraft belasten, sondern hauptsächlich die Vulnerabilitäten des migrantischen Ankommens ausreizen. Dass das Ankommen selbst eine fundamentale Vulnerabilität ist und weitere Vulnerabilitäten nach sich zieht, kann in verschiedenen Szenarien des Ankommens von GastarbeiterInnen (Höhne et al. 2014), von Geflüchteten (Kukovetz 2017, Huke 2020), von ,Illegalisierten (Karakayali 2008) oder vom „just-in-time“ und „tothe-point" zugestellten Personal (Bojadžijev et al. 2018) nachvollzogen werden. Spätkapitalistische Ausbeutung mag bei Ankunft ausschließlich und am härtesten MigrantInnen treffen, sie erntet dennoch eine andere Reaktion als die klassische proletarische Antwort darauf. Im proletarischen Klassenkampfnarrativ exemplifiziert Ausbeutung das Problem von Kapitalismus schlechthin, weil durch Ausbeutung Menschen trotz Anstrengungen und Arbeitsleistung unten an der gesellschaftlichen Ungleichheitsordnung platziert und gehalten werden, für die eingesteckten pains viel zu wenige gains erhalten, um von unten abzuheben und ihre Lage zu verbessern (Wright 2005). Bei migrantischen bottom hoppers und bottom comers kommt hingegen die beim Ankommen auf sie wartende Ausbeutung spätkapitalistischer Prägung anders und unter dem verkehrten Vorzeichen an, nicht nur als der Fluch, sich an der Gesellschaft unten anhängen zu müssen, sondern auch und vor allem als die Chance, sich unten anhängen zu können. ${ }^{23}$ Wie auch ungezähmt, gezielt auf MigrantInnen und auf die Vulnerabilitäten des migrantischen Ankommens gerichtet, dient die Ausbeutung beim Ankommen jener, die als MigrantInnen gelten, als eine Art Haken zum Anhän-

23 Was keine generelle Abneigung gegen proletarischen Klassenkampf nach sich ziehen muss, uns dennoch auf andere Kampfarenen, Arten von Kampf und vom Kampfverhalten von unten hellhörig machen muss, die dem Handlungsrepertoire von „everyday resistance“ (Scott 1990) und von „social nonmovements“ (Bayat 2010) näher sind. gen, wenn auch von unten. ${ }^{24}$ So ein Ankommen bringt MigrantInnen in den schwer lösbaren Widerspruch, mit dem Akt der Migration kapitalistische Verhältnisse der postkolonialen Abkoppelung von Herkunftsarenen zurückzuweisen, durch das bottom hoppen gegen Kapitalismus aufzubegehren, um durch ebendiesen Kraftakt des bottom hoppen die Anschlussfindung an Topadressen von Kapitalismus vorzunehmen. Ziehen wir auch die Ankunft, nicht nur die Flucht, in Betracht, erweist sich das „Begehren der Migration“ (Tsianos/Karakayali 2012: 18$)^{25}$ als widersprüchliches, zerreißendes Begehren - gegen Kapitalismus aufzubegehren und nach Kapitalismus zu begehren, Kapitalismus zu entkommen und im Kapitalismus anzukommen, Kapitalismus $z u$ widerstehen und Kapitalismus unwiderstehlich $\mathrm{zu}$ finden.

Soziostrukturell unten sein - weil es ja Unten ist, wo MigrantInnen Fuß fassen - und zugleich bei Ankunft auf einer höheren Gangschaltung spätkapitalistischer Ausbeutung unterwegs zu sein, die widersprüchliche Rolle zu spielen, Kapitalismus von unten anzutreiben und zu betreiben, nicht nur von Kapitalismus und Ausbeutung bei Ankunft betroffen zu sein, sondern selbst durch Kapitalismus und Ausbeutung gute Treffer zum Ankommen zu landen, ist der Widerspruch, der migrantisches Ankommen schwer fassbar oder gar unfassbar für die geläufigen politischen, sozialtheoretischen, medialen Bildgebungen der westlichen Ankunftsgesellschaften der Gegenwart macht.

Durch den Paradigmenwechsel und die Fokusverlegung von Wanderung aufs Ankommen öffnen sich neue, kritische Vistas ${ }^{26}$ auf Migration und Migrationsgesellschaft, die allesamt um das Ankommen von Menschen von unten zentriert sind und die Frage, was in der Migrationsgesellschaft los ist, auf den Punkt bringen: ,Aufs Ankommen kommt es an!

Die Gesellschaften an den Top-Adressen der liberal-demokratischen und kapitalistischen Welt der Gegenwart werden durch ein und dieselbe kritische und realistische Perspektive als Migrationsgesellschaft oder postmigrantische Gesellschaft erfasst und durch

24 Zur Erinnerung: Als MigrantInnen gelten Menschen, die der Drift postkolonialer Abkoppelung entkommen und den Anschluss an die Spitzenbühnen von Kapitalismus, Liberalismus und Demokratie durch Wandern und Ankommen von unten versuchen.

25 „Flucht“ vor „miserablen ausbeuterischen Existenzbedingungen“ (Hess 2016: 58) zu „begehren“ (Tsianos/ Karakayali 2012: 18).

26 Siehe oben, Fußnote 11.
IIIOMENTUM QUARTERLY IIIIIIIIIIIIIIIIIII 115 
beide diagnostischen Konzepte in letzter Konsequenz als die Ankunftsgesellschaft anvisiert. In der Ankunftsgesellschaft wird das Gesellschaftsleben, die Struktur und Dynamik der Gesellschaft vor allem durch jenes bewegte und bewegende Ankommen von unten geprägt, das nach der Wanderung stattfindet und PostMigration ist. Es ist das Ankommen, in dem Millionen gleichzeitig und am selben Ort Fuß fassen, dabei millionenfach Ungleichheiten durchleben, Widersprüche hochwirbeln, Polarisationen freisetzen und den neuen Boden zum Beben bringen.

\section{Literatur:}

Anderson, B./Keith, M. (Hg., 2014): Migration. The COMPAS Anthology. Oxford: Compas.

Bastian, M. (2014): Time. In: Anderson, B./Keith, M. (Hg.): Migration: The COMPAS Anthology. Oxford: Compas.

Bauböck, R. (Hg., 2006): Migration and Citizenship. Legal Status, Rights and Political Participation. IMISCOE Reports. Amsterdam: Amsterdam University Press.

Bayat, A. (2010): Life as Politics. How Ordinary People Change the Middle East. Amsterdam: University Press.

Bojadžijev, M. (2018): Migration und Integration. Zur Genealogie des zentralen Dispositivs in der Migrationsgesellschaft. Migration und Soziale Arbeit, 40 (1), 54. Online: https://transversal.at/pdf/journal-text/1673/ [22.06.2021]

Bojadžijev, M./Römhild, R. (2014): Was kommt nach dem „transnational turn“? Perspektiven für eine kritische Migrationsforschung. In: Labor Migration (Hg.): Vom Rand ins Zentrum. Perspektiven einer kritischen Migrationsforschung. Berlin: Panama Verlag, 10-25.

Bojadžijev, M./Mezzadra, S./Altenried, M./Höfler, L./Wallis, M. (2018): Migration und Logistik. Politiken und Vermittlung mobiler Arbeit (Forschungsbericht des Berliner Instituts für empirische Integrations- und Migrationsforschung (BIM), Humboldt-Universität zu Berlin im Rahmen des Forschungs-Interventions-Clusters „Solidarität im Wandel?") Berlin: Humboldt-Universität zu Berlin.

bordermonitoring.eu (2015): Entlang der Balkanroute. Online: https://bordermonitoring.eu/balkan/2015/10/entlang-der-balkanroute/ [24.05.2021]

Castles, S./Miller, M. J. (1998): The Age of Migration. International Population Movements in the Modern World. London: MacMillan Press.

Castro Varela, M. do Mar/Mecheril, P. (2011): Migration. In: Arndt, S./Ofuatey-Alazard, N. (Hg.): Wie Rassismus aus Wörtern spricht. (K)Erben des Kolonialismus im Wissensarchiv deutsche Sprache. Ein kritisches Nachschlagewerk. Münster: Unrast, 154-177.
Chakrabarty, D. (1992): Postcoloniality and the Artifice of History: „Who Speaks for ,Indian' Pasts?“ Representations, 37 (Winter 1992), 1-26.

Chakrabarty, D. (2000): Provincializing Europe: Postcolonial Thought and Historical Difference. Princeton: Princeton University Press.

Espahangizi, K./Hess, S./Karakayali, J./Kasparek, B./Pagano, S./Rodatz, S./Tsianos, V. (2016): Rassismus in der postmigrantischen Gesellschaft. Zur Einleitung. In: Espahangizi, K./Hess, S./Karakayali, J./Kasparek, B./Pagano, S./Rodatz, S./Tsianos, V. (Hg.): Rassismus in der postmigrantischen Gesellschaft. movements. Journal für kritische Migrations- und Grenzregimeforschung, 2 (1), 9-23.

Faist, Th. (2006): The Transnational Social Spaces of Migration. Working Papers - Center on Migration, Citizenship and Development, Nr. 10.

Fischer, O. I. (2020): Geflüchtetenprotest und Gewerkschaften. Verhandlungen von Repräsentation im deutschen Arbeitsund Migrationsregime. Bielefeld: Transcript.

Foroutan, N./Karakayali, J./Spielhaus, R. (2018): Postmigrantische Perspektiven. Ordnungssysteme, Repräsentationen, Kritik. Frankfurt a. M.: Campus Verlag.

Foroutan, N. (2019): Die postmigrantische Gesellschaft. Ein Versprechen der pluralen Demokratie. Bielefeld: Transcript.

Foroutan, N./Ikiz, D. (2016): Migrationsgesellschaft. In: Mecheril, P./Kourabas, V./Rangger, M. (Hg.): Handbuch Migrationspädagogik. Weinheim/Basel: Beltz, 138-151.

Fraser, N. (2019): Die Krise der Demokratie: Über politische Widersprüche des Finanzmarktkapitalismus jenseits des Politizismus. In: Dörre, K./Fraser. N./Lessenich, S./Rosa, H.: Was stimmt nicht mit der Demokratie? Berlin: Suhrkamp, 77-100.

Gans, H. J. (2009): First generation decline: downward mobility among refugees and immigrants. Ethnic and Racial Studies, 32 (9), 1658-1670.

Ginzburg, C. (1995): Spurensicherung. Die Wissenschaft auf der Suche nach sich selbst. Berlin: Wagenbach.

Griffiths, M./Rogers, A./Anderson, A. (2013): Migration, Time and Temporalities: Review and Prospect. COMPAS Research Resources Paper, March 2013, University of Oxford.

Harvey, D. (2005): A Brief History of Neoliberalism. Oxford: Oxford University Press.

Hess, S./Kasparek, B. (Hg., 2010): Grenzregime. Diskurse, Praktiken, Institutionen in Europa. Berlin: Assoziation A.

Hess, S./Kasparek, B./Kron, S./Rodatz, M./Schwertl, M./Sontowski, S. (Hg., 2016): Der lange Sommer der Migration. Grenzregime III. Berlin: Assoziation A.

Hess, S. (2016): Migration als widerständige Praxis - Die Autonomie der Migration als theoretische Intervention in die border studies. In: Tietje, O. (Hg.): Bedrohte Subjektivitäten. Böckler Stiftung, 54-67.

Hill, M./Yıldız, E. (2019): Postmigrantische Visionen. Erfahrungen, Ideen, Reflexionen. Bielefeld: Transcript. 
Hoerder, D. (2014): Global Migration. In: Anderson, B./Keith M. (Hg.): Migration: The COMPAS Anthology. Oxford: Compas.

Höhne, J./Linden, B./Seils, E./Wiebel, A. (2014): Die Gastarbeiter. Geschichte und aktuelle soziale Lage. WSI Report. September 2014, 1-29.

Huke, N. (2020): Ganz Unten in der Hierarchie. Rassismus als Arbeitsmarkthindernis für Geflüchtete. Eine Studie des durch das Bundesministerium für Bildung und Forschung (BMBF) geförderten Verbundprojekts „Willkommenskultur und Demokratie in Deutschland“. Erschienen im Dezember 2020.

Hummrich, M./Terstegen, M. (2020): Migration: Begriffsbestimmungen und pädagogische Diskurse. Eine Einführung. Wiesbaden: Springer.

International Organization for Migration (IOM 2019a): Glossary on Migration. International Migration Law No. 34, Geneva: IOM. Online: www.iom.int/glossary-migration-2019 [16.05.2021].

International Organization for Migration (IOM 2019b): World Migration Report 2020. Geneva: IOM.

Kaloianov, R. (2019): Das Migrantische: Underclass in the Making und Kritik. In: Böttcher, A./Hill, M./Rotter, A./ Schacht, F./Wolf, M. A./Yildı, E. (Hg.): Migration bewegt und bildet. Kontrapunktische Betrachtungen. Innsbruck: Innsbruck University Press, 147-169.

Karaca, C. (2010): Es kamen Menschen an [Lied]. Retrieved from: https://www.youtube.com/ watch? $\mathrm{v}=\mathrm{uLrFnXQn} 2 \mathrm{Og}$ [01.05. 2020].

Karakayali, S. (2008): Gespenster der Migration. Zur Genealogie illegaler Einwanderung in der Bundesrepublik Deutschland. Bielefeld: Transcript.

Kelly, E./Dobbin, F. (2001): How Affirmative Action Became Diversity Management. Employers Response to Antidiscrimination Law, 1961-1996. In: Skretny, J. D. (Hg.): Color Lines. Affirmative Action, Immigration and Civil Rights Options for America. Chicago: University of Chicago Press, 87-118.

Knaut, A. (Hg., 2013): Biometrische Identitäten und ihre Rolle in den Diskursen um Sicherheit und Grenzen. Eine Tagungsdokumentation. Arbeitsgruppe Informatik in Bildung und Gesellschaft, Institut für Informatik, Humboldt-Universität zu Berlin, August 2013.

Kukovetz, B. (2017): Irreguläre Leben. Handlungspraxen zwischen Abschiebung und Niederlassung. Bielefeld: Transcript

Milanovic, B. (2016): Global Inequality: A New Approach for the Age of Globalization. Cambridge, MA: Harvard University Press.

Mecheril, P. (2004): Einführung in die Migrationspädagogik. Weinheim/Basel: Beltz.

Mecheril, P. (2016): Migrationspädagogik - ein Projekt. In: Mecheril, P./Kourabas, V./Rangger, M. (Hg.): Handbuch Migrationspädagogik. Weinheim/Basel: Beltz, 8-32.
Mezzadra, S./Neilson, B. (2013): Border as Method, or, the Multiplication of Labor. Durham/London: Duke University Press.

Mezzadra, S. (2020): Abolitionist vistas of the human. Border struggles, migration and freedom of movement. Citizenship Studies, 24 (4), 424-440.

Oberlechner, M. (2018): Zur Aktualität kritisch-theoretischen Denkens für die neue Lehramtsausbildung in Österreich: Ein diversitätssensibler Entwurf inklusiver Migrationspädagogik. Momentum Quarterly. Zeitschrift für Sozialen Fortschritt, 7 (4), 221-232.

OECD (2018): Working Together for Local Integration of Migrants and Refugees. Paris: OECD Publishing. Online: http://dx.doi.org/10.1787/9789264085350-en [16.06.2021].

$\mathrm{OECD/EU} \mathrm{(2018):} \mathrm{Settling} \mathrm{In} \mathrm{2018.} \mathrm{Indicators} \mathrm{of} \mathrm{Immigrant} \mathrm{In-}$ tegration. Paris: OECD Publishing \& Brussels: European Union. Online: https://doi.org/10.1787/9789264307216en [16.06.2021].

Otto, H.-U./Schrödter, M. (2006): Soziale Arbeit in der Migrationsgesellschaft. Neue Praxis. 8 [Sonderheft]

Papademetriou, D. G./Somerville, W./Sumption, M. (2009): The Social Mobility of Immigrants and Their Children. Migration Policy Institute, June 2009.

Rahner, S. (2014): Fachkräftebedarf und Zuwanderung: Geschichte und Perspektiven. Aus Politik und Zeitgeschichte, 61 (43), 29-35.

Römhild, R. (2009): Aus der Perspektive der Migration. Die Kosmopolitisierung Europas. In: Hess, S./Binder, J./ Moser, J. (Hg.): No Integration?! Kulturwissenschaftliche Beiträge zur Integrationsdebatte in Europa. Bielefeld: Transcript, 225-239.

Samaddar, R. (2018): The Postcolonial Age of Migration. London/New York: Routledge.

Sassen, S. (2017): Migranten, Siedler, Flüchtlinge: Von der Massenauswanderung zur Festung Europa. Fischer Digital.

Schwiertz, H./Ratfisch, P. (2015): Antimigrantische Politik und der „Sommer der Migration“. In: Rosa-LuxemburgStiftung (Hg.): Analysen, 25, 4-23.

Scott, J. (1990): Domination and the Arts of Resistance. New Haven, CT: Yale University Press.

Shachar, A. (2009): The Birthright Lottery: Citizenship and Global Inequality. Cambridge MA: Harvard University Press.

Schiller, N.G. (2018): Theorising Transnational Migration in Our Times: A multiscalar temporal perspective. Nordic Journal of Migration Research, 8 (4), 201-212.

Tazzioli, M. (2019): The Making of Migration: The Biopolitics of Mobility at Europe's Borders. London: Sage Publications.

Treibel, A. (2008): Migration in modernen Gesellschaften: soziale Folgen von Einwanderung, Gastarbeit und Flucht. Weinheim/München: Juventa.

Tsianos, V./Karakayali, S. (2012): Die frohe Botschaft der Autonomie der Migration. Annäherung an einen umstrittenen Begriff. Prager Frühling. Magazin für Freiheit und Sozialismus, 10, 18-21.
IIIOMENTUM QUARTERLY "IIIIIIIIIIIIIIIIII!

117 
United Nations Department of Economic and Social Affairs (UN DESA) 1998: Recommendations on Statistics of International Migration: Revision 1. United Nations, New York. Online: https://unstats.un.org/unsd/publication/ SeriesM/SeriesM_58rev1E.pdf [16.05.2021].

Wallerstein, I. (2019): Welt-System-Analyse. Eine Einführung. Herausgegeben und übersetzt von Julien Bucher, Felix Merz und Sylke Nissen. Wiesbaden: Springer VS, (EBook), https://doi.org/10.1007/978-3-658-21962-8 .

World Bank (2017): Monitoring Global Poverty: Report of the Commission on Global Poverty. Washington, DC: World Bank.

Wright, E. O. (2005): Foundations of a neo-Marxist class analysis. In: Wright, E. O. (Hg.): Approaches to Class Analysis. Cambridge: Cambridge University Press.

Ylldız, E. (2014): Postmigrantische Perspektiven. In: Ylldiz, E./Hill, M. (Hg.): Nach der Migration. Postmigrantische Perspektiven jenseits der Parallelgesellschaft. Bielefeld: Transcript, 20-35.

Yıldız, E./Hill, M. (Hg., 2014): Nach der Migration. Postmigrantische Perspektiven jenseits der Parallelgesellschaft. Bielefeld: Trans 